\title{
SOME ESTIMATES ON EXPONENTIALS OF SOLUTIONS TO STOCHASTIC DIFFERENTIAL EQUATIONS
}

\author{
JIONGMIN YONG
}

Received 26 March 2004 and in revised form 25 August 2004

Exponential of functionals of solutions to certain stochastic differential equations (SDEs) plays an interesting role in some mathematical finance problems. The purpose of this paper is to establish some estimates for these exponentials.

\section{Introduction}

We begin with a couple of motivations. To this end, let $\left(\Omega, \mathscr{F}_{F},\left\{\mathscr{F}_{t}\right\}_{t \geq 0}, \mathbf{P}\right)$ be a complete filtered probability space on which a $d$-dimensional standard Brownian motion $W(\cdot)$ is defined with $\left\{\mathscr{F}_{t}\right\}_{t \geq 0}$ being its natural filtration augmented by all the P-null sets. Consider the Black-Scholes market model (see $[11,13])$

$$
\begin{aligned}
& d P_{0}(t)=P_{0}(t) r(t) d t, \\
& d P_{i}(t)=P_{i}(t)\left[b_{i}(t) d t+\left\langle\sigma_{i}(t), d W(t)\right\rangle\right], \quad 1 \leq i \leq n,
\end{aligned}
$$

where $P_{0}(\cdot)$ and $P_{i}(\cdot)$ are the price processes of the bond and the $i$ th stock, respectively, $r(\cdot)$ is the interest rate of the bond, and $b_{i}(\cdot)$ and $\sigma_{i}(\cdot)$ are appreciation rate and the volatility (vector) of the $i$ th stock, respectively. Suppose an investor has an initial wealth $y$ and he/she is taking self-financing trading strategies. Then the wealth process $Y(\cdot)$ satisfies the following stochastic differential equation (SDE, for short) (see [18]):

$$
\begin{gathered}
d Y(t)=[r(t) Y(t)+\langle b(t)-r(t) \mathbf{1}, \pi(t)\rangle] d t+\langle\pi(t), \sigma(t) d W(t)\rangle, \\
Y(0)=y
\end{gathered}
$$

where $\pi(\cdot) \equiv\left(\pi_{1}(\cdot), \ldots, \pi_{n}(\cdot)\right)^{T}$ with $\pi_{i}(t)$ being the market value of the $i$ th stock held by the investor at time $t, b(\cdot)=\left(b_{1}(\cdot), \ldots, b_{n}(\cdot)\right)^{T}, \sigma(\cdot)=\left(\sigma_{1}(\cdot), \ldots, \sigma_{n}(\cdot)\right)$, and $\mathbf{1} \equiv(1, \ldots$, $1)^{T} \in \mathbb{R}^{n}$. [8])

Now, suppose the short interest rate $r(\cdot)$ satisfies the general Hull-White model (see

$$
\begin{gathered}
d r(t)=[\alpha(t)-\beta(t) r(t)] d t+r(t)^{\delta}\langle\nu(t), d W(t)\rangle, \\
r(0)=r_{0},
\end{gathered}
$$


where $\alpha, \beta:[0, \infty) \rightarrow(0, \infty), v:[0, \infty) \rightarrow \mathbb{R}^{d}$ are given (deterministic) maps, $\delta \in[0,1]$, and $r_{0}>0$. When $d=1$, the case $\delta=0$ is called (generalized) Vasicek's model (see [17]) and $\delta=1 / 2$ is called (generalized) Cox-Ingersoll-Ross (CIR, for short) model (see [3] and also [13]). It is known that when $\delta=0$ or $\delta \in[1 / 2,1]$, for any $r_{0}>0,(1.3)$ admits a unique strong solution $r(\cdot)$ (see $[9,10]$ ). It is also known that for $d=1$ and $\delta \in(0,1 / 2)$, when $\alpha(\cdot)=\beta(\cdot)=0$ and $\sigma(t) \equiv 1,(1.3)$ does not have a strong solution, and it seems to be unknown if $\alpha(\cdot)$ and $\beta(\cdot)$ are nonzero (and $d=1, \delta \in(0,1 / 2)$ ), see, for example, the comment in [19, page 82]. We point out here that any strong solution $r(\cdot)$ of $(1.3)$ is unbounded in general.

We return to (1.2). Formally, the solution of (1.2) is given by

$$
\begin{aligned}
Y(t) \equiv Y(t ; y, \pi(\cdot))=e^{\int_{0}^{t} r(u) d u}[ & y+\int_{0}^{t} e^{-\int_{0}^{s} r(u) d u}\langle b(s)-r(s) 1, \pi(s)\rangle d s \\
& \left.+\int_{0}^{t} e^{-\int_{0}^{t} r(u) d u}\langle\pi(s), \sigma(s) d W(s)\rangle\right] .
\end{aligned}
$$

In studying some mathematical finance problems such as contingent claim pricing, optimal investment, and so on, one hopes that the wealth process $Y(\cdot)$ is a well-defined process belonging to, say, $L_{\mathscr{F}}^{1}(\Omega ; C([0, T] ; \mathbb{R}))$, the set of all continuous $\left\{\mathscr{F}_{t}\right\}_{t \geq 0}$-adapted processes $\varphi(\cdot)$ such that $E\left[\sup _{t \in[0, T]}|Y(t)|\right]<\infty$ or $E\left[Y(T)^{\lambda}\right]<\infty$, for some $\lambda \in(0,1)$, at least. On the other hand, in almost all relevant studies, we should at least be allowed to take $\pi(\cdot)=0$ (which means that the investor puts all the wealth in the bond and does not hold any stocks). Then, in order that $Y(\cdot ; y, 0) \in L_{\mathscr{F}}^{1}(\Omega ; C([0, T] ; \mathbb{R}))$ or $E\left[Y(T ; y, 0)^{\lambda}\right]<\infty$, one should at least have

$$
E\left[e^{\lambda \int_{0}^{T} r(u) d u}\right]<\infty,
$$

for some $\lambda>0$. In the case that $r(\cdot)$ is bounded, (1.5) holds automatically. However, when $r(\cdot)$ is a strong solution of (1.3), it is by no means clear whether (1.5) holds. As a matter of fact, unfortunately, we will show the following result.

Propsition 1.1. When $\delta>1 / 2$, (1.5) fails for any $T>0$ and $\lambda>0$. In the case $\delta=1 / 2$, there exists a $T_{0}$ (depending on $\lambda>0$ ) such that (1.5) fails when $T>T_{0}$.

The above result tells us that in studying problems that involve $E\left[Y(T ; y, \pi(\cdot))^{\lambda}\right]$, the interest rate model (1.3) with $\delta>1 / 2$ is not very suitable, and the CIR model could be used but one has to restrict the time duration $T$.

Next, we look at another interesting problem. We still take market model (1.1) and the wealth process equation (1.2). We assume that $n=d$ and $\sigma(t)^{-1}$ exists for all $t \in[0, T]$. Then one can define

$$
\theta(t)=\sigma(t)^{-1}[b(t)-r(t) \mathbf{1}], \quad t \in[0, T]
$$

which is referred to as the risk premium of the market. It is known that if the so-called Novikov's condition (see $[10,14]$ ) holds,

$$
E\left[e^{(1 / 2) \int_{0}^{T}|\theta(t)|^{2} d t}\right]<\infty,
$$


or the so-called Kazamaki's condition (see [12]) (which is weaker than (1.7)) holds,

$$
E\left[e^{(1 / 2) \int_{0}^{t}\langle\theta(s), d W(s)\rangle}\right]<\infty, \quad \forall t \in[0, T]
$$

then the process

$$
M(t ; \theta(\cdot)) \triangleq e^{f_{0}^{t}(\theta(s), d W(s)\rangle-(1 / 2) \int_{0}^{t}|\theta(s)|^{2} d s}, \quad t \in[0, T],
$$

is a uniformly integrable $\left\{\mathscr{F}_{t}\right\}_{t \geq 0}$-martingale on $[0, T]$, and

$$
\widetilde{W}(t) \triangleq W(t)-\int_{0}^{t} \theta(s) d s, \quad t \in[0, T]
$$

is an $\left\{\mathscr{F}_{t}\right\}_{t \geq 0}$-standard Brownian motion on $\left(\Omega, \mathscr{F}_{T}, \widetilde{\mathbf{P}}_{T}\right)$ with $\widetilde{\mathbf{P}}_{T}$ being a probability measure on $\left(\Omega, \mathscr{F}_{T}\right)$ equivalent to $\left.\mathbf{P}\right|_{\mathscr{F}_{T}}$, defined by

$$
d \widetilde{\mathbf{P}}_{T}=M(T ; \theta(\cdot)) d \mathbf{P} .
$$

Moreover, one calls $\widetilde{\mathbf{P}}_{T}$ an equivalent martingale measure of the market, and every discounted stock price process is an $\left\{\mathscr{F}_{t}\right\}_{t \geq 0}$-martingale under $\widetilde{\mathbf{P}}_{T}$. In this case, the market is arbitrage-free. One usually refers to (1.9), (1.10), and (1.11) as Girsanov's transformation (see [10]).

In mathematical finance, the existence of equivalent martingale measure plays a very important role because it is (almost) equivalent to the market being arbitrage-free (see [4]). Thus, people hope that either (1.7) or (1.8) holds. Again, when $r(\cdot)$ is a strong solution of (1.3), say, it is unbounded in general. In such a case, even if $\sigma(\cdot)^{-1}$ is bounded, we do not have the boundedness of $\theta(\cdot)$. Hence, one would like to know when (1.7) and (1.8) hold. The following result will be proved in a later section.

Propsition 1.2. Let $n=d=1$ and let $b(\cdot), \sigma(\cdot)$, and $\sigma(\cdot)^{-1}$ be bounded. Let $\theta(\cdot)$ be defined by (1.6) with $r(\cdot)$ being the strong solution of (1.3). Then (1.7) fails as long as $\delta \geq$ $1 / 2$, and for $\delta=0$, there exists a $T_{0}$ such that (1.7) fails as well if $T>T_{0}$. Also, (1.8) fails when $\delta \geq 1 / 2$ together with some additional conditions.

The above is again a kind of negative result, which tells us that in the context involving equivalent martingale measures and/or arbitrage-freeness, one has to be careful to use (1.3) as the short-interest rate model. This remark also applies to the context when a multi-factor model is used (see [1]). The situation for more general models found in $[5,6]$ is still under careful investigation and we will address the results elsewhere.

The above two motivations suggest we formulate a more general problem. Consider the following $\operatorname{SDE}$ on $\left(\Omega, \mathscr{F}_{t},\left\{\mathscr{F}_{t}\right\}_{t \geq 0}, \mathbf{P}\right)$ :

$$
\begin{gathered}
d X(t)=b(t, X(t)) d t+\sigma(t, X(t)) d W(t), \quad t \in[0, \infty), \\
X(0)=X_{0}
\end{gathered}
$$


where $b:[0, \infty) \times \mathbb{R}^{n} \rightarrow \mathbb{R}^{n}, \sigma:[0, \infty) \times \mathbb{R}^{n} \rightarrow \mathbb{R}^{n \times d}$ are given maps. Suppose (1.12) admits a strong solution $X(\cdot)$. Next, let $\varphi, \psi: \mathbb{R}^{n} \rightarrow[0, \infty)$ and $\mu: \mathbb{R}^{n} \rightarrow \mathbb{R}^{d}$ be given. We pose the following problem.

Problem (E). Find conditions on $b(\cdot), \sigma(\cdot), \varphi(\cdot), \psi(\cdot)$, and $\mu(\cdot)$ such that the following hold:

$$
\begin{gathered}
E\left[\sup _{t \in[0, T]} e^{\varphi(X(t))}\right]<\infty, \quad T \in[0, \infty), \\
E\left[e^{\int_{0}^{T} \psi(X(s)) d s}\right]<\infty, \quad T \in[0, \infty), \\
E\left[\sup _{t \in[0, T]} e^{\int_{0}^{t}\langle\mu(X(s)), d W(s)\rangle}\right]<\infty, \quad T \in[0, \infty) .
\end{gathered}
$$

It is standard that under some mild conditions, such as uniform Lipschitz condition on the coefficients $b(t, x)$ and $\sigma(t, x)$ in $x$, or even some weaker conditions (see $[9,10])$, (1.12) admits a unique strong solution $X(\cdot)$. Moreover, when $b(t, x)$ and $\sigma(t, x)$ grow at most linearly in $x$, the following estimate holds for the solution $X(\cdot)$ : for any $m>0$ and $T>0$,

$$
E\left[\sup _{t \in[0, T]}|X(t)|^{m}\right] \leq C_{m, T}\left(1+\left|X_{0}\right|^{m}\right), \quad \forall(t, x) \in[0, T] \times \mathbb{R}^{n}
$$

for some constant $C_{m, T}>0$ depending on $m, T$, and $b(\cdot)$ and $\sigma(\cdot)$. It is then very natural to ask when (1.13) holds. From this point of view, one sees that our problem has its own interest, which is concerned with estimates of solutions to SDEs. We would like to point out that an interesting case for (1.13) is $\varphi(x)=|x|^{\gamma}$ for some $\gamma>0$. This then indicates that our problem is also closely related to the topics discussed in [2].

We would also like to mention the book [19] in which the exponential functional of Brownian motion (or even a Lévy process) has been systematically studied. If one replaces the Brownian motion by the strong solution of some (nonlinear) SDE, then one faces the problem that we are going to study in this paper. Hence, this work is closely related to that of [19] in a certain sense as well.

The rest of the paper is organized as follows. In Section 2, we present some preliminary results. Section 3 is devoted to some estimates for stochastic differential inequalities. In Section 4, we give precise statements and proofs of Propositions 1.1 and 1.2. Finally, general results for Problem $(E)$ are presented in Section 5.

\section{Some preliminaries}

In this section, we present some preliminary results. First of all, we introduce some spaces. For any Euclidean space $H$ (such as $\mathbb{R}^{n}, \mathbb{R}^{n \times m}$, etc.) whose norm is denoted by $|\cdot|$, 
we define

$$
\begin{aligned}
L_{\mathscr{F}}^{p}\left(\Omega ; L^{q}(0, T ; H)\right) \triangleq & \left\{\eta:[0, T] \times \Omega \longrightarrow H \mid \eta(\cdot) \text { is }\left\{\mathscr{F}_{t}\right\}_{t \geq 0}\right. \text {-adapted, } \\
& \left.E\left[\int_{0}^{T}|\eta(t)|^{q} d t\right]^{p / q}<\infty\right\}, \quad 1 \leq p, q<\infty, \\
L_{\mathscr{F}}^{q}\left(0, T ; L^{p}(\Omega ; H)\right) \triangleq\left\{\eta:[0, T] \times \Omega \longrightarrow H \mid \eta(\cdot) \text { is }\left\{\mathscr{F}_{t}\right\}_{t \geq 0}\right. \text {-adapted, } & \\
& \left.\int_{0}^{T}\left[E|\eta(t)|^{p}\right]^{q / p} d t<\infty\right\}, \quad 1 \leq p, q<\infty .
\end{aligned}
$$

The spaces of the above types corresponding to $p=\infty$ and/or $q=\infty$ can be defined in an obvious way. We will simply denote $L_{\mathscr{F}}^{p}\left(0, T ; L^{p}(\Omega ; H)\right)=L_{\mathscr{F}}^{p}\left(\Omega ; L^{p}(0, T ; H)\right)=L_{\mathscr{F}}^{p}(0, T ; H)$, for any $1 \leq p \leq \infty$. Also, we define

$$
\begin{aligned}
& L_{\mathscr{F}}^{p}(\Omega ; C([0, T] ; H)) \\
& \triangleq\left\{\eta:[0, T] \times \Omega \longrightarrow H \mid \eta(\cdot) \text { is }\left\{\mathscr{F}_{t}\right\}_{t \geq 0}\right. \text {-adapted with continuous paths, } \\
& \left.\quad E\left[\sup _{t \in[0, T]}|\eta(t)|^{p}\right]<\infty\right\}, \quad 1 \leq p<\infty, \\
& C_{\mathscr{F}}\left([0, T] ; L^{q}(\Omega ; H)\right) \\
& \triangleq\left\{\eta:[0, T] \times \Omega \longrightarrow H \mid \eta(\cdot) \text { is }\left\{\mathscr{F}_{t}\right\}_{t \geq 0}\right. \text {-adapted with continuous paths, } \\
& \left.\quad \sup _{t \in[0, T]} E|\eta(t)|^{q}<\infty\right\}, \quad 1 \leq q<\infty .
\end{aligned}
$$

We now present the following result.

Lemma 2.1. Let $X(\cdot)$ be defined as follows:

$$
X(t)=\int_{0}^{t} \sigma(s) d W(s), \quad t \in[0, \infty)
$$

with $\sigma(\cdot) \in L_{\mathscr{F}}^{1}\left(\Omega ; L^{2}\left(0, T ; \mathbb{R}^{n \times d}\right)\right)$ for each $T>0$.

(i) Suppose, for some nonnegative deterministic function $\delta_{0}(\cdot) \in L_{\text {loc }}^{2}(0, \infty)$,

$$
\left|\sigma(t, \omega)^{T} x\right| \geq \delta_{0}(t)|x|, \quad \forall t \in[0, \infty), x \in \mathbb{R}^{n} \text {, a.s. } \omega \in \Omega .
$$

Then

$$
E\left[e^{|X(t)|^{\nu}}\right]=\infty
$$


provided

$$
\begin{aligned}
& \text { either } \quad \gamma \in(2, \infty), \quad \int_{0}^{t} \delta_{0}(s)^{2} d s>0, \\
& \text { or } \quad \gamma=2, \quad 2 \int_{0}^{t} \delta_{0}(s)^{2} d s \geq 1 .
\end{aligned}
$$

(ii) Suppose, for some nonnegative deterministic function $L_{0}(\cdot) \in L_{\mathrm{loc}}^{2}(0, \infty)$,

$$
|\sigma(t, \omega)| \leq L_{0}(t), \quad \forall t \in[0, \infty), \text { a.s. } \omega \in \Omega \text {. }
$$

Then

$$
E\left[\sup _{t \in[0, T]} e^{|X(t)|^{\gamma}}\right]<\infty
$$

provided

$$
\begin{aligned}
& \text { either } \quad \gamma \in[0,2) \\
& \text { or } \quad \gamma=2, \quad 2 \int_{0}^{T} L_{0}(s)^{2} d s<1 .
\end{aligned}
$$

Proof. (i) By Itô's formula and induction, making use of (2.4), we have

$$
E\left[|X(t)|^{2 k}\right] \geq \frac{(2 k) !}{2^{k} k !}\left(\int_{0}^{t} \delta_{0}(s)^{2} d s\right)^{k}, \quad t \in[0, \infty) .
$$

Let $\ell_{m}$ be the unique integer such that $\gamma m \geq 2 \ell_{m}>\gamma m-2$. Then it follows that (recalling Stirling's formula $\lim _{m \rightarrow \infty} m ! e^{m} / m^{m} \sqrt{2 \pi m}=1$ )

$$
\begin{aligned}
E\left[e^{|X(t)|^{\gamma}}\right] & =\sum_{m=0}^{\infty} \frac{E\left[|X(t)|^{\gamma m}\right]}{m !} \geq \sum_{m=0}^{\infty} \frac{\left\{E\left[|X(t)|^{2 \ell_{m}}\right]\right\}^{\gamma m / 2 \ell_{m}}}{m !} \\
& \geq \sum_{m=0}^{\infty} \frac{1}{m !}\left[\frac{\left(2 \ell_{m}\right) !}{2^{\ell_{m}} \ell_{m} !}\left(\int_{0}^{t} \delta_{0}(s)^{2} d s\right)^{\ell_{m}}\right]^{\gamma m / 2 \ell_{m}} \\
& =\sum_{m=0}^{\infty} \frac{1}{m !}\left(\frac{1}{2} \int_{0}^{t} \delta_{0}(s)^{2} d s\right)^{\gamma m / 2}\left[\frac{\left(2 \ell_{m}\right) !}{\ell_{m} !}\right]^{\gamma m / 2 \ell_{m}} \\
& \sim 1+\sum_{m=1}^{\infty} \frac{e^{m}}{m^{m} \sqrt{2 \pi m}}\left(\frac{1}{2} \int_{0}^{t} \delta_{0}(s)^{2} d s\right)^{\gamma m / 2}\left[\frac{\left(2 \ell_{m}\right)^{2 \ell_{m}} e^{\ell_{m}} \sqrt{4 \pi \ell_{m}}}{\ell_{m}^{\ell_{m}} e^{2 \ell_{m}} \sqrt{2 \pi \ell_{m}}}\right]^{\gamma m / 2 \ell_{m}} \\
& \leq 1+\sum_{m=1}^{\infty} \frac{m^{((\gamma-2) / 2) m}}{\sqrt{\pi m}}\left(\gamma \int_{0}^{t} \delta_{0}(s)^{2} d s\right)^{\gamma m / 2}\left(1-\frac{2}{\gamma m}\right)^{\gamma m / 2-\gamma) / 2} .
\end{aligned}
$$


Clearly, when $\gamma>2$ and $\int_{0}^{t} \delta_{0}(s)^{2} d s>0$, the right-hand side of (2.11) diverges. For the case $\gamma=2$, one has

$$
\begin{aligned}
E\left[e^{|X(t)|^{2}}\right] & =\sum_{m=0}^{\infty} \frac{E\left[|X(t)|^{2 m}\right]}{m !} \geq \sum_{m=0}^{\infty} \frac{1}{m !} \frac{(2 m) !}{2^{m} m !}\left(\int_{0}^{t} \delta_{0}(s)^{2} d s\right)^{m} \\
& \sim 1+\sum_{m=1}^{\infty}\left(\frac{1}{2} \int_{0}^{t} \delta_{0}(s)^{2} d s\right)^{m} \frac{(2 m)^{2 m} e^{2 m} \sqrt{4 \pi m}}{m^{2 m} e^{2 m} 2 \pi m} \\
& =1+\sum_{m=1}^{\infty} \frac{1}{\sqrt{\pi m}}\left(2 \int_{0}^{t} \delta_{0}(s)^{2} d s\right)^{m} .
\end{aligned}
$$

The above diverges if $2 \int_{0}^{t} \delta_{0}(s)^{2} d s \geq 1$. This proves (i).

(ii) By (2.7), similar to (2.10), we are able to show that

$$
E\left[|X(t)|^{2 k}\right] \leq \frac{(2 k) !}{2^{k} k !}\left(\int_{0}^{t} L_{0}(s)^{2} d s\right)^{k}, \quad t \in[0, \infty), k \geq 1
$$

Then it follows from Doob's inequality that

$$
E\left[\sup _{t \in[0, T]}|X(t)|^{2 k}\right] \leq\left(\frac{2 k}{2 k-1}\right)^{2 k} \frac{(2 k) !}{2^{k} k !}\left(\int_{0}^{T} L_{0}(s)^{2} d s\right)^{k}, \quad T \in[0, \infty), k \geq 1 .
$$

Now, for $\gamma>0$, let $k_{m} \geq 1$ be the unique integer such that $\gamma m \leq 2 k_{m}<\gamma m+2$. Then it follows that (similar to (2.11))

$$
\begin{aligned}
& E\left[\sup _{t \in[0, T]} e^{|X(t)|^{\gamma}}\right] \\
& =\sum_{m=0}^{\infty} \frac{E\left[\sup _{t \in[0, T]}|X(t)|^{\gamma m}\right]}{m !} \\
& \leq \sum_{m=0}^{\infty} \frac{\left\{E\left[\sup _{t \in[0, T]}|X(t)|^{2 k_{m}}\right]\right\}^{\gamma m / 2 k_{m}}}{m !} \\
& \leq \sum_{m=0}^{\infty} \frac{1}{m !}\left[\left(\frac{2 k_{m}}{2 k_{m}-1}\right)^{2 k_{m}} \frac{\left(2 k_{m}\right) !}{2^{k_{m}} k_{m} !}\left(\int_{0}^{t} L_{0}(s)^{2} d s\right)^{k_{m}}\right]^{\gamma m / 2 k_{m}} \\
& \sim 1+\sum_{m=1}^{\infty} \frac{e^{m}\left(2 k_{m} /\left(2 k_{m}-1\right)\right)^{\gamma m}}{m^{m} \sqrt{2 \pi m}}\left(\frac{1}{2} \int_{0}^{t} L_{0}(s)^{2} d s\right)^{\gamma m / 2}\left[\frac{\left(2 k_{m}\right)^{2 k_{m}} e^{k_{m}} \sqrt{4 \pi k_{m}}}{k_{m}^{k_{m}} e^{2 k_{m}} \sqrt{2 \pi k_{m}}}\right]^{\gamma m / 2 k_{m}} \\
& \leq 1+\sum_{m=1}^{\infty} \frac{2^{\gamma m}}{m^{((2-\gamma) / 2) m} \sqrt{\pi m}}\left(\int_{0}^{t} L_{0}(s)^{2} d s\right)^{\gamma m / 2}\left(\gamma+\frac{2}{m}\right)^{\gamma m / 2} e^{((2-\gamma) / 2) m} .
\end{aligned}
$$


When $\gamma \in[0,2)$, the right-hand side of $(2.15)$ converges. In the case $\gamma=2$, we have

$$
\begin{aligned}
E\left[\sup _{t \in[0, T]} e^{|X(t)|^{2}}\right] & =\sum_{m=0}^{\infty} \frac{E\left[\sup _{t \in[0, T]}|X(t)|^{2 m}\right]}{m !} \\
& \leq 1+\sum_{m=1}^{\infty} \frac{1}{m !}\left(\frac{2 m}{2 m-1}\right)^{2 m} \frac{(2 m) !}{2^{m} m !}\left(\int_{0}^{t} L_{0}(s)^{2} d s\right)^{m} \\
& \sim 1+\sum_{m=1}^{\infty}\left(\frac{2 m^{2}}{(2 m-1)^{2}} \int_{0}^{t} L_{0}(s)^{2} d s\right)^{m} \frac{(2 m)^{2 m} e^{2 m} \sqrt{4 \pi m}}{m^{2 m} e^{2 m} 2 \pi m} \\
& =1+\sum_{m=1}^{\infty} \frac{1}{\sqrt{\pi m}}\left(\frac{2}{(1-1 / 2 m)^{2}} \int_{0}^{t} L_{0}(s)^{2} d s\right)^{m} .
\end{aligned}
$$

The right-hand side of (2.16) converges if $2 \int_{0}^{t} L_{0}(s)^{2} d s<1$, proving (ii).

The above result leads to the following corollary for the exponentials involving standard Brownian motions.

Corollary 2.2. Let $W(\cdot)$ be a d-dimensional standard Brownian motion and $\lambda>0$. Then

$$
\begin{gathered}
E\left[e^{\lambda|W(t)|^{\gamma}}\right]=\infty, \quad \gamma \in(2, \infty), t \in(0, \infty) \quad \text { or } \quad \gamma=2, t \in\left[\frac{1}{2 \lambda}, \infty\right) \\
E\left[\sup _{t \in[0, T]} e^{\lambda|W(t)|^{\gamma}}\right]<\infty, \quad \gamma \in[0,2), t \in[0, \infty) \quad \text { or } \quad \gamma=2, t \in\left[0, \frac{1}{2 \lambda}\right) .
\end{gathered}
$$

Proof. The case $\gamma=0$ is obvious. Thus, we only need to consider the case $\gamma>0$. Let $\sigma(t) \equiv$ $\lambda^{1 / \gamma} I$ in (2.3) with $n=d$. Then, applying Lemma 2.1, we obtain (2.17) for the case $\gamma \neq 2$. For the case $\gamma=2$, we have

$$
\begin{aligned}
E\left[e^{\lambda|W(t)|^{2}}\right] & =\left\{E\left[e^{\lambda\left|W_{1}(t)\right|^{2}}\right]\right\}^{d} \\
& =\left\{\sum_{m=0}^{\infty} \frac{\lambda^{m} E\left[\left|W_{1}(t)\right|^{2 m}\right]}{m !}\right\}^{d}=\left\{\sum_{m=0}^{\infty} \frac{1}{m !} \frac{(2 m) !}{m !}\left(\frac{\lambda t}{2}\right)^{m}\right\}^{d} \\
& \sim\left\{1+\sum_{m=1}^{\infty} \frac{(2 m)^{2 m} e^{2 m} \sqrt{4 \pi m}}{m^{2 m} e^{2 m} 2 \pi m}\left(\frac{\lambda t}{2}\right)^{m}\right\}^{d}=\left\{1+\sum_{m=1}^{\infty} \frac{(2 \lambda t)^{m}}{\sqrt{\pi m}}\right\}^{d} .
\end{aligned}
$$

Then we obtain the conclusions for $\gamma=2$.

We make a remark here. For $n=d=1$, it is known by Dambis-Dubins-Schwarz theo$\operatorname{rem}[11,15,16]$ that when $\sigma(\cdot) \in L_{\mathscr{F}}^{2}(0, T ; \mathbb{R})$, for each $T>0$, we may define

$$
\begin{gathered}
\tau(s)=\inf \left\{t \geq\left. 0\left|\int_{0}^{t}\right| \sigma(u)\right|^{2} d u>s\right\}, \\
B(s)=\int_{0}^{\tau(s)} \sigma(u) d W(u) \equiv X(\tau(s)), \\
\mathscr{G}_{s} \triangleq \mathscr{F}_{\tau(s)}, \quad s \geq 0 .
\end{gathered}
$$


Then $B(\cdot)$ is a one-dimensional standard Brownian motion under filtration $\left\{\mathscr{G}_{s}\right\}_{s \geq 0}$. Moreover,

$$
X(t)=\int_{0}^{t} \sigma(u) d W(u)=B\left(\int_{0}^{t}|\sigma(u)|^{2} d u\right), \quad t \geq 0
$$

Consequently, for any $T>0$, if we define $T^{\prime}=\int_{0}^{T} L_{0}(s)^{2} d s$, then, for any $\gamma \in[0,2)$,

$$
E\left[\sup _{t \in[0, T]} e^{|X(t)|^{\gamma}}\right]=E\left[\sup _{t \in[0, T]} e^{\left|B\left(\int_{0}^{t}|\sigma(s)|^{2} d s\right)\right|^{\gamma}}\right] \leq E\left[\sup _{t \in\left[0, T^{\prime}\right]} e^{|B(t)|^{\gamma}}\right]<\infty
$$

provided that we have first proved (2.17), for which a careful estimate is no simpler than (2.10), (2.11), and (2.12). On the other hand, if $n, d \geq 2$, a direct application of DambisDubins-Schwarz theorem might still be possible, but it will be much messier. Further, to prove the conclusion for $\gamma>2$, such an approach seems not any simpler than (2.14) and (2.15). Hence, we have taken a direct (and elementary) approach without using DambisDubins-Schwarz theorem in the above.

Note that (2.4) implies $\sigma(\cdot) \in L_{\mathscr{F}}^{2}\left(0, T ; L^{\infty}\left(\Omega ; \mathbb{R}^{n \times d}\right)\right)$. In this case, one may actually take

$$
L_{0}(t)=\underset{\omega \in \Omega}{\operatorname{ess} \sup }|\sigma(t, \omega)|, \quad t \geq 0
$$

We point out that if $\sigma(\cdot) \notin L_{\mathscr{F}}^{2}\left(0, T ; L^{\infty}\left(\Omega ; \mathbb{R}^{n \times d}\right)\right)$, (2.8) might fail for $\gamma \in(0,2)$. In fact, for $n=d=1$, we take $\sigma(t)=W(t)$. Then

$$
\sigma(\cdot) \in\left\{\bigcap_{p \geq 1} C_{\mathscr{F}}\left([0, T] ; L^{p}(\Omega ; \mathbb{R})\right)\right\} \backslash L_{\mathscr{F}}^{1}\left(0, T ; L^{\infty}(\Omega ; \mathbb{R})\right), \quad \forall T>0 .
$$

For such a case, by Itô's formula, we have

$$
E\left[e^{|X(T)|}\right]=E\left[e^{\left|\int_{0}^{T} W(t) d W(t)\right|}\right] \geq E\left[e^{\int_{0}^{T} W(t) d W(t)}\right]=e^{-T / 2} E\left[e^{(1 / 2) W(T)^{2}}\right]=\infty,
$$

provided $T \geq 1$, by Corollary 2.2. This means that, in some sense, condition (2.7) is sharp. Also, we note that for part (i), we need only $(2.4)$, and $\sigma(\cdot) \in L_{\mathscr{F}}^{2}\left(0, T ; L^{\infty}\left(\Omega ; \mathbb{R}^{n \times d}\right)\right)$ is not needed.

\section{Estimates for scalar stochastic differential inequalities}

In this section, we establish some exponential estimates for solutions to some scalar stochastic differential inequalities which will be useful below.

Theorem 3.1. Let $\xi_{0} \in \mathbb{R}$ and let $b_{0}, b_{1}:[0, \infty) \times \Omega \rightarrow \mathbb{R}$ and $\sigma:[0, \infty) \times \Omega \rightarrow \mathbb{R}^{d}$ be $\left\{\mathscr{F}_{t}\right\}_{t \geq 0}$-adapted processes satisfying

$$
\begin{array}{cc}
b_{0}(\cdot) \in L_{\mathscr{F}}^{\infty}\left(\Omega ; L^{1}(0, T ; \mathbb{R})\right), \quad b_{1}(\cdot) \in L_{\mathscr{F}}^{\infty}(0, T ; \mathbb{R}), & \forall T>0 . \\
\sigma(\cdot) \in L_{\mathscr{F}}^{2}\left(0, T ; L^{\infty}\left(\Omega ; \mathbb{R}^{d}\right)\right), &
\end{array}
$$


296 Estimates on exponentials of solutions to SDEs

(i) Let $\xi(\cdot)$ be an $\left\{\mathscr{F}_{t}\right\}_{t \geq 0}$-adapted process satisfying

$$
\begin{gathered}
d \xi(t) \geq\left[b_{0}(t)+b_{1}(t) \xi(t)\right] d t+\langle\sigma(t), d W(t)\rangle, \quad t \geq 0, \\
\xi(0)=\xi_{0} .
\end{gathered}
$$

Suppose $\varphi: \mathbb{R} \rightarrow[0, \infty)$ is continuous such that for some $\gamma \geq 2$ and $c>0$,

$$
\varliminf_{x \rightarrow \infty} \frac{\varphi(x)}{x^{\gamma}}>c
$$

Then

$$
E\left[e^{\varphi\left(\xi(T)^{+}\right)}\right]=\infty
$$

provided

$$
\begin{aligned}
& \text { either } \quad \gamma>2, \quad \int_{0}^{T} \underset{\omega \in \Omega}{\operatorname{essinf}}|\sigma(s, \omega)|^{2} d s>0, \\
& \text { or } \quad \gamma=2, \quad 2 c\left[\underset{\omega \in \Omega}{\operatorname{essinf}} e^{2 \int_{0}^{T} b_{1}(u, \omega) d u}\right] \int_{0}^{T} \underset{\omega \in \Omega}{\operatorname{essinf}} e^{-2 \int_{0}^{s} b_{1}(u, \omega) d u}|\sigma(s, \omega)|^{2} d s>1 .
\end{aligned}
$$

Further, if $b_{1}(\cdot)$ is deterministic, then

$$
E\left[e^{\int_{0}^{T} \varphi\left(\xi(t)^{+}\right) d t}\right]=\infty
$$

provided

$$
\begin{aligned}
& \text { either } \quad \gamma>2, \quad \int_{0}^{T} \underset{\omega \in \Omega}{\operatorname{essinf}}|\sigma(s, \omega)|^{2} d s>0, \\
& \text { or } \quad \gamma=2, \quad \frac{2 c}{T} \int_{0}^{T}\left(\int_{s}^{T} e^{\int_{s}^{t} b_{1}(u) d u} d t\right)^{2} \underset{\omega \in \Omega}{\operatorname{essinf}}|\sigma(s, \omega)|^{2} d s>1 .
\end{aligned}
$$

In addition, for the case $\gamma=2$, if $\xi_{0} \geq 0$ and $b_{0}(\cdot)$ is nonnegative valued, then " $>$ " in the second lines in (3.5) and (3.7) can be replaced by " $\geq$ ".

(ii) Let $\xi(\cdot)$ be an $\left\{\mathscr{F}_{t}\right\}_{t \geq 0}$-adapted process satisfying

$$
\begin{gathered}
d \xi(t) \leq\left[b_{0}(t)+b_{1}(t) \xi(t)\right] d t+\langle\sigma(t), d W(t)\rangle, \quad t \geq 0, \\
\xi(0)=\xi_{0} .
\end{gathered}
$$

Suppose $\varphi: \mathbb{R} \rightarrow[0, \infty)$ is continuous such that for some $\gamma \in[0,2]$ and $c>0$,

$$
\varlimsup_{x \rightarrow \infty} \frac{\varphi(x)}{x^{\gamma}}<c
$$

Then

$$
E\left[\sup _{t \in[0, T]} e^{\varphi\left(\xi(t)^{+}\right)}\right]<\infty
$$


provided

$$
\begin{aligned}
& \text { either } \quad y \in[0,2) \text {, } \\
& \text { or } \quad \gamma=2, \quad 2 c\left[\operatorname{essup}_{(t, \omega) \in[0, T] \times \Omega} e^{2 \int_{0}^{t} b_{1}(u, \omega) d u}\right] \int_{0}^{T} \operatorname{esssup}_{\omega \in \Omega} e^{-2 \int_{0}^{s} b_{1}(u, \omega) d u}|\sigma(s, \omega)|^{2} d s<1 .
\end{aligned}
$$

Further,

$$
E\left[e^{\int_{0}^{T} \varphi\left(\xi(t)^{+}\right) d t}\right]<\infty
$$

provided

$$
\begin{aligned}
& \text { either } \quad \gamma \in[0,2) \text {, } \\
& \text { or } \quad \gamma=2, \quad 2 T c\left[\underset{(t, \omega) \in[0, T] \times \Omega}{\operatorname{ess} \sup } e^{2 \int_{0}^{t} b_{1}(u, \omega) d u}\right] \int_{0}^{T} \underset{\omega \in \Omega}{\operatorname{esssup}} e^{-2 \int_{0}^{s} b(u, \omega) d u}|\sigma(s, \omega)|^{2} d s<1 .
\end{aligned}
$$

In the above, (3.2) and (3.8) are understood as corresponding integral inequalities (involving Lebesgue and Itô integrals). Thus, the process $\xi(\cdot)$ involved in (3.2) or (3.8) is not necessarily an Itô process (i.e., it is not necessarily a solution to an SDE). The same convention applies below as well.

Proof. (i) By (3.2), we have

$$
\begin{aligned}
\xi(t) & \geq e^{\int_{0}^{t} b_{1}(u) d u}\left[\xi_{0}+\int_{0}^{t} e^{-\int_{0}^{s} b_{1}(u) d u} b_{0}(s) d s+\int_{0}^{t} e^{-\int_{0}^{s} b_{1}(u) d u}\langle\sigma(s), d W(s)\rangle\right] \\
& \equiv c_{0}(t) \int_{0}^{t}\langle\hat{\sigma}(s), d W(s)\rangle+f_{0}(t), \quad t \geq 0
\end{aligned}
$$

where

$$
\begin{gathered}
c_{0}(t)=e^{\int_{0}^{t} b_{1}(u) d u}, \quad \hat{\sigma}(t)=e^{-\int_{0}^{t} b_{1}(u) d u} \sigma(t), \\
f_{0}(t)=\underset{\omega \in \Omega}{\operatorname{essinf}}\left\{e^{\int_{0}^{t} b_{1}(u) d u}\left[\xi_{0}+\int_{0}^{t} e^{-\int_{0}^{s} b_{1}(u) d u} b_{0}(s) d s\right]\right\}, \quad t \geq 0 .
\end{gathered}
$$

By (3.3), we see that there exists an $R>0$ such that

$$
\varphi(x) \geq c x^{\gamma}-R, \quad \forall x \in \mathbb{R} .
$$

Note that for $\gamma \geq 2$ and $\mu \in(0,1)$,

$$
\left[(a+b)^{+}\right]^{\gamma} \geq \mu^{\gamma-1}\left[a^{+}\right]^{\gamma}-\left(\frac{\mu}{1-\mu}\right)^{\gamma-1}\left[(-b)^{+}\right]^{\gamma}, \quad \forall a, b \in \mathbb{R} .
$$


Hence (we denote $\bar{c}_{0}(t)=\operatorname{essinf}_{\omega \in \Omega} c_{0}(t)$ )

$$
\begin{aligned}
E\left[e^{\varphi\left(\xi(t)^{+}\right)}\right] & \geq e^{-R} E\left[e^{c\left|\xi(t)^{+}\right| \gamma}\right] \\
& \geq e^{-R} E\left[e^{c\left|\left[c_{0}(t) \int_{0}^{t}\langle\hat{\sigma}(s), d W(s)\rangle+f_{0}(t)\right]^{+}\right| \gamma}\right] \\
& \geq e^{-R-\mu^{\gamma-1}(1-\mu)^{1-\gamma}\left|\left[-f_{0}(t)\right]^{+}\right| \gamma^{\gamma}} E\left[e^{\mu^{\gamma-1} c \bar{c}_{0}(t)^{\gamma}\left|\left[\int_{0}^{t}\langle\hat{\sigma}(s), d W(s)\rangle\right]^{+}\right| \gamma}\right] \\
& \geq \frac{1}{2} e^{-R-\mu^{\gamma-1}(1-\mu)^{1-\gamma}\left|\left[-f_{0}(t)\right]^{+}\right| \gamma} E\left[e^{\mu^{\gamma^{-1}} c \bar{c}_{0}(t)^{\gamma}\left|\int_{0}^{t}\langle\hat{\sigma}(s), d W(s)\rangle\right|^{\gamma}}\right]=\infty
\end{aligned}
$$

provided

$$
\gamma>2, \quad \int_{0}^{t} \underset{\omega \in \Omega}{\operatorname{essinf}}|\hat{\sigma}(s, \omega)|^{2} d s>0,
$$

which is equivalent to the first line in (3.5). In the case $\gamma=2,(3.18)$ becomes

$$
\begin{aligned}
E\left[e^{\varphi\left(\xi(t)^{+}\right)}\right] & \geq e^{-R} E\left[e^{c\left|\xi(t)^{+}\right|^{2}}\right] \\
& \geq \frac{1}{2} e^{-R-\mu(1-\mu)^{-1}\left|\left[-f_{0}(t)\right]^{+}\right|^{2}} E\left[e^{\mu c \bar{c}_{0}(t)^{2}\left|\int_{0}^{t}\langle\hat{\sigma}(s), d W(s)\rangle\right|^{2}}\right]=\infty
\end{aligned}
$$

provided

$$
2 \mu c \int_{0}^{t} \bar{c}_{0}(t)^{2} \underset{\omega \in \Omega}{\operatorname{essinf}}|\hat{\sigma}(s, \omega)|^{2} d s \geq 1
$$

which is implied by the second line in (3.5) when $1-\mu>0$ is small enough.

We now prove (3.6) under condition (3.7). By (3.16), (3.17), and Jensen's inequality, we have

$$
\begin{aligned}
\int_{0}^{T} \varphi\left(\xi(t)^{+}\right) d t & \geq c \int_{0}^{T}\left|\left[\xi(t)^{+}\right]\right|^{\gamma} d t-R T \\
& =c T \int_{0}^{T}\left[\xi(t)^{+}\right]^{\gamma} \frac{1}{T} d t-R T \\
& \geq c T\left[\left(\frac{1}{T} \int_{0}^{T} \xi(t) d t\right)^{+}\right]^{\gamma}-R T \\
& =c T^{1-\gamma}\left[\left(\int_{0}^{T}\left[c_{0}(t) \int_{0}^{t}\langle\hat{\sigma}(s), d W(s)\rangle+f_{0}(t)\right] d t\right)^{+}\right]^{\gamma}-R T \\
& \geq c T^{1-\gamma}\left\{\mu^{\gamma-1}\left[\left(\int_{0}^{T}\left[c_{0}(t) \int_{0}^{t}\langle\hat{\sigma}(s), d W(s)\rangle\right] d t\right)^{+}\right]^{\gamma}\right. \\
& \geq\left[\left(\int _ { 0 } ^ { T } \left\langle\left[c^{1 / \gamma}\left(\frac{\mu}{T}\right)^{\gamma-1}\left[\left(-\int_{0}^{T} f_{0}(t) d t\right)^{+}\right]^{\gamma}\right\}-R T\right.\right.\right. \\
& {\left.\left.\left.\left[c_{0}(t) d t\right] \hat{\sigma}(s), d W(s)\right\rangle\right)^{+}\right]^{\gamma}-C_{\mu} . }
\end{aligned}
$$


Hence,

$$
\begin{aligned}
E\left[e^{\int_{0}^{T} \varphi\left(\xi(t)^{+}\right) d t}\right] & \geq e^{-C_{\mu}} E\left[e^{\left[\left(\int_{0}^{T}\left\langle\left[c^{1 / \gamma}(\mu / T)^{(\gamma-1) / \gamma} \int_{s}^{T} c_{0}(t) d t\right] \hat{\sigma}(s), d W(s)\right\rangle\right)^{+}\right]^{\gamma}}\right] \\
& =\frac{1}{2} e^{-C_{\mu}} E\left[e^{\left.\iint_{0}^{T}\left\langle\left[c^{1 / \gamma}(\mu / T)^{(\gamma-1) / \gamma} \int_{s}^{T} c_{0}(t) d t\right] \hat{\sigma}(s), d W(s)\right\rangle d t\right|^{\gamma}}\right]=\infty
\end{aligned}
$$

provided $\gamma>2$ and

$$
\int_{0}^{T}\left(\int_{s}^{T} c_{0}(t) d t\right)^{2} \underset{\omega \in \Omega}{\operatorname{essinf}}|\hat{\sigma}(s, \omega)|^{2} d s>0,
$$

which is equivalent to the first line in (3.7). In the case $\gamma=2,(3.23)$ becomes

$$
E\left[e^{\int_{0}^{T} \varphi\left(\xi(t)^{+}\right) d t}\right] \geq \frac{1}{2} e^{-C_{\mu}} E\left[e^{\left|\int_{0}^{T}\left\langle\left[c^{1 / 2}(\mu / T)^{1 / 2} \int_{s}^{T} c_{0}(t) d t\right] \hat{\sigma}(s), d W(s)\right\rangle d t\right|^{2}}\right]=\infty
$$

provided

$$
\frac{2 c \mu}{T} \int_{0}^{T}\left(\int_{s}^{T} c_{0}(t) d t\right)^{2} \underset{\omega \in \Omega}{\operatorname{essinf}}|\hat{\sigma}(s, \omega)|^{2} d s \geq 1,
$$

which is implied by the second line in (3.7) when $1-\mu>0$ is small enough.

Now, for $\gamma=2$, if $\xi_{0} \geq 0$ and $b_{0}(\cdot)$ is nonnegative valued, we may take $f_{0}(t) \equiv 0$. Consequently, (3.17) is not necessary here. Then, instead of (3.20), we have

$$
E\left[e^{\varphi\left(\xi(t)^{+}\right)}\right] \geq e^{-R} E\left[e^{c\left|\xi(t)^{+}\right|^{2}}\right] \geq \frac{1}{2} e^{-R} E\left[e^{c \bar{c}_{0}(t)^{2}\left|\int_{0}^{t}\langle\hat{\sigma}(s), d W(s)\rangle\right|^{2}}\right]=\infty,
$$

provided (3.21) holds with $\mu=1$, and (3.25) becomes

$$
E\left[e^{\int_{0}^{T} \varphi\left(\xi(t)^{+}\right) d t}\right] \geq \frac{1}{2} e^{-R T} E\left[e^{\left|\int_{0}^{T}\left\langle\left[c^{1 / 2} T^{-1 / 2} \int_{s}^{T} c_{0}(t) d t\right] \hat{\sigma}(s), d W(s)\right\rangle d t\right|^{2}}\right]=\infty
$$

provided (3.26) holds with $\mu=1$.

(ii) By (3.8), we have

$$
\begin{aligned}
\xi(t) \leq & e^{\int_{0}^{t} b_{1}(u) d u} \xi_{0}+e^{\int_{0}^{t} b_{1}(u) d u} \int_{0}^{t} e^{-\int_{0}^{s} b_{1}(u) d u} b_{0}(s) d s \\
& +e^{\int_{0}^{t} b_{1}(u) d u} \int_{0}^{t} e^{-\int_{0}^{s} b_{1}(u) d u}\langle\sigma(s), d W(s)\rangle \\
\leq & c_{0}(t) \int_{0}^{t}\langle\hat{\sigma}(t), d W(t)\rangle+\bar{f}_{0}(t),
\end{aligned}
$$

where $c_{0}(\cdot), \hat{\sigma}(\cdot)$ are the same as in (3.15) and

$$
\bar{f}_{0}(t)=\operatorname{esssup}_{\omega \in \Omega}\left[e^{\int_{0}^{t} b_{1}(u) d u}\left(\xi_{0}+\int_{0}^{t} e^{-\int_{0}^{s} b_{1}(u) d u} b_{0}(s) d s\right)\right] .
$$

By (3.9), there exists an $R>0$ such that

$$
\varphi(x) \leq c x^{\gamma}+R, \quad \forall x \in \mathbb{R} .
$$


Without loss of generality, we may assume that $\gamma \in(1,2]$, otherwise (i.e., $\gamma \in[0,1)$ ), we could replace $\gamma$ in (3.31) by $\bar{\gamma}=\gamma \vee 3 / 2$. Next, we note that for any martingale $\eta(\cdot)$, with each $\eta(t)$ being normal, one has (note Doob's inequality)

$$
\begin{aligned}
E\left[\sup _{t \in[0, T]} e^{\left[\eta(t)^{+}\right]^{\gamma}}\right] & \leq \sum_{m=0}^{\infty} \frac{1}{m !} E\left[\sup _{t \in[0, T]}\left[\eta(t)^{+}\right]^{\gamma m}\right] \\
& \leq 1+\sum_{m=1}^{\infty} \frac{1}{m !}\left(\frac{\gamma m}{\gamma m-1}\right)^{\gamma m} E\left\{\left[\eta(T)^{+}\right]^{\gamma m}\right\} \\
& \leq 1+\frac{e \gamma}{2(\gamma-1)} \sum_{m=1}^{\infty} \frac{1}{m !} E\left\{|\eta(T)|^{\gamma m}\right\} \leq \frac{e \gamma}{2(\gamma-1)} E\left[e^{\mid \eta(T)^{\mid \gamma}}\right] .
\end{aligned}
$$

Here, we have used the fact that $(\gamma m /(\gamma m-1))^{\gamma m} \leq e \gamma /(\gamma-1)$. Hence, by Lemma 2.1 (letting $\left.\widehat{c}_{0}(T)=\operatorname{ess} \sup _{(t, \omega) \in[0, T] \times \Omega} c_{0}(t)\right)$, one has

$$
\begin{aligned}
& E\left[\sup _{t \in[0, T]} e^{\varphi\left(\xi(t)^{+}\right)}\right] \leq e^{R} E\left[\sup _{t \in[0, T]} e^{c\left[\xi(t)^{+}\right]^{\nu}}\right] \\
& \leq e^{R} E\left[\sup _{t \in[0, T]} e^{c\left|\left[c_{0}(t) \int_{0}^{t}\langle\hat{\sigma}(s), d W(s)\rangle+\bar{f}_{0}(t)\right]^{+}\right| \nu}\right] \\
& \leq e^{R+\sup _{t \in[0, T]} 2^{(\gamma-1)^{+}}\left|\bar{f}_{0}(t)^{+}\right| \gamma} E\left[\sup _{t \in[0, T]} e^{2^{\gamma-1} c||\left[c_{0}(t) \int_{0}^{t}\langle\hat{\sigma}(s), d W(s)\rangle\right]^{+} \mid \gamma}\right] \\
& \leq C E\left[\sup _{t \in[0, T]} e^{\left|\left[2^{(y-1) / \gamma} c^{1 / \gamma} c_{0}(t) \int_{0}^{t}\langle\hat{\sigma}(s), d W(s)\rangle\right]^{+}\right| y}\right] \\
& \leq C E\left[\sup _{t \in[0, T]} e^{\left|2^{2(\gamma-1) / \gamma} c^{1 / \gamma} \hat{c}_{0}(T) \int_{0}^{t}\langle\hat{\sigma}(s), d W(s)\rangle\right|^{\nu}}\right]<\infty
\end{aligned}
$$

provided $\gamma<2$. In the case $\gamma=2$, for any $\varepsilon \in(0,1)$, similar to the above, one has

$$
\begin{aligned}
& E\left[\sup _{t \in[0, T]} e^{\varphi\left(\xi(t)^{+}\right)}\right] \leq e^{R} E\left[\sup _{t \in[0, T]} e^{c\left[\xi(t)^{+}\right]^{2}}\right] \\
& \leq e^{R} E\left[\sup _{t \in[0, T]} e^{c\left|\left[c_{0}(t) \int_{0}^{t}\langle\hat{\sigma}(s), d W(s)\rangle+\bar{f}_{0}(t)\right]^{+}\right|^{2}}\right] \\
& \leq e^{R+\sup _{t \in[0, T]}(1+1 / \mathcal{\varepsilon})\left|\hat{f}_{0}(t)^{+}\right|^{2}} E\left[\sup _{t \in[0, T]} e^{(1+\varepsilon) c\left|\left[c_{0}(t) \int_{0}^{t}\langle\hat{\sigma}(s), d W(s)\rangle\right]^{+}\right|^{2}}\right] \\
& \leq C_{\varepsilon} E\left[\sup _{t \in[0, T]} e^{\left|\left[(1+\varepsilon)^{1 / 2} c^{1 / 2} c_{0}(t) \int_{0}^{t}\langle\hat{\sigma}(s), d W(s)\rangle\right]^{+}\right|^{2}}\right] \\
& \leq C_{\varepsilon} E\left[\sup _{t \in[0, T]} e^{\left|(1+\varepsilon)^{1 / 2} c^{1 / 2} \hat{c}_{0}(T) \int_{0}^{t}\langle\hat{\sigma}(s), d W(s)\rangle\right|^{2}}\right]<\infty
\end{aligned}
$$


provided

$$
2(1+\varepsilon) \hat{c}_{0}(T)^{2} \int_{0}^{T} \underset{\omega \in \Omega}{\operatorname{esssup}}|\hat{\sigma}(s)|^{2} d s<1,
$$

which is implied by the second line in (3.11) by taking $\varepsilon>0$ small enough.

Finally, we prove (3.12) under condition (3.13). When $\gamma \in[0,2)$, by what we have just proved, one has

$$
E\left[e^{e_{0}^{T} \varphi\left(\xi(t)^{+}\right) d t}\right] \leq E\left[e^{T \sup _{t \in[0, T]} \varphi\left(\xi(t)^{+}\right)}\right]=E\left[\sup _{t \in[0, T]} e^{T \varphi\left(\xi(t)^{+}\right)}\right]<\infty .
$$

In the case $\gamma=2$, we use (3.10) with $\varphi(\cdot)$ replaced by $T \varphi(\cdot)$ and $c$ by $T c$. This completes the proof.

When all the coefficients are deterministic, condition (3.5) can be replaced by

$$
\begin{aligned}
& \text { either } \quad \gamma>2, \quad \int_{0}^{T}|\sigma(s)|^{2} d s>0, \\
& \text { or } \quad \gamma=2, \quad 2 c \int_{0}^{T} e^{2 \int_{s}^{T} b_{1}(u) d u}|\sigma(s)|^{2} d s>1,
\end{aligned}
$$

and (3.11) can be replaced by

$$
\begin{aligned}
& \text { either } \quad y \in[0,2) \text {, } \\
& \text { or } \quad \gamma=2, \quad 2 c\left[\sup _{t \in[0, T]} e^{2 \int_{0}^{t} b_{1}(u) d u}\right] \int_{0}^{T} e^{-2 \int_{0}^{s} b_{1}(u) d u}|\sigma(s)|^{2} d s<1 .
\end{aligned}
$$

Further, if $b_{1}(\cdot)$ is nonnegative valued, then the second line in (3.38) is equivalent to the following:

$$
2 c \int_{0}^{T} e^{2 \int_{s}^{T} b_{1}(u) d u}|\sigma(s)|^{2} d s<1 .
$$

From (3.37) and (3.39), we see that this part of our result is sharp in some sense. Similarly, in the case that all the coefficients are deterministic, (3.7) and (3.13) can be replaced, respectively, by the following

$$
\begin{aligned}
& \text { either } \quad y>2, \quad \int_{0}^{T}|\sigma(s)|^{2} d s>0, \\
& \text { or } \quad \gamma=2, \quad \frac{2 c}{T} \int_{0}^{T}\left(\int_{s}^{T} e^{\int_{s}^{t} b_{1}(u) d u} d t\right)^{2}|\sigma(s)|^{2} d s>1 ; \\
& \text { either } \quad \gamma \in[0,2), \\
& \text { or } \quad \gamma=2, \quad 2 T c\left[\sup _{t \in[0, T]} e^{2 \int_{0}^{t} b_{1}(u) d u}\right] \int_{0}^{T} e^{-2 \int_{0}^{s} b_{1}(u) d u}|\sigma(s)|^{2} d s<1 .
\end{aligned}
$$


In the case that $b_{1}(\cdot)$ is nonnegative (and deterministic) valued, the inequality in the second line in (3.41) is equivalent to

$$
2 T c \int_{0}^{T} e^{2 \int_{s}^{T} b_{1}(u) d u}|\sigma(s)|^{2} d s<1 .
$$

On the other hand, when $b_{1}(\cdot)$ is nonnegative (and deterministic) valued, one has

$$
\frac{2 c}{T} \int_{0}^{T}\left(\int_{s}^{T} e^{\int_{s}^{t} b_{1}(u) d u} d t\right)^{2}|\sigma(s)|^{2} d s \leq 2 T c \int_{0}^{T} e^{2 \int_{s}^{T} b_{1}(u) d u}|\sigma(s)|^{2} d s
$$

and the inequality is strict if $b_{1}(\cdot)$ and $|\sigma(\cdot)|$ are strictly positive. Thus, there is a gap between conditions (3.40) and (3.41). Next, we note that when $T>0$ small enough, the second lines in (3.11) and (3.13) hold, and when $T>0$ large enough, the second lines in (3.5) and (3.7) hold, provided $|\sigma(\cdot)|$ is bounded from below and $b_{1}(\cdot)$ is bounded.

\section{Estimates involving interest rate term structure models}

In this section, we will give precise statements as well as proofs of Propositions 1.1 and 1.2. To this end, we make the following assumption.

$(\mathrm{H} 1)$ Let $\alpha, \beta:[0, \infty) \rightarrow(0, \infty), v:[0, \infty) \rightarrow \mathbb{R}^{d}$ be deterministic maps such that $\alpha(\cdot) \in$ $L_{\mathrm{loc}}^{1}(0, \infty), \beta(\cdot) \in L_{\mathrm{loc}}^{\infty}(0, \infty)$, and $\nu(\cdot) \in L_{\mathrm{loc}}^{2}\left(0, \infty ; \mathbb{R}^{d}\right)$.

Before stating and proving the results of this section, we make some remarks. As we mentioned in the introduction, under (H1), the SDE (1.3) admits a unique strong solution for $\delta=0$ or $d \in[1 / 2,1]$, whereas the case $\delta \in(0,1 / 2)$ with $\alpha(\cdot)$ and $\beta(\cdot)$ nonzero is not well understood (see [9]). Thus, the results below will only take care of the cases $\delta=0$ and $\delta \in[1 / 2,1]$. Further, we recall that for the case $\delta \in[1 / 2,1]$, the strong solution $r(\cdot)$ to $(1.3)$ is nonnegative valued, but could take negative values for $\delta=0, r(\cdot)$.

Now, we make Proposition 1.1 precise.

Theorem 4.1. Let (H1) hold, $\delta=0$ or $\delta \in[1 / 2,1]$. Let $r(\cdot)$ be the strong solution of $(1.3)$. Then, for $\lambda>0$,

$$
E\left[e^{\lambda \int_{0}^{T} r(t) d t}\right]=\infty
$$

provided

$$
\begin{aligned}
& \text { either } \delta \in\left(\frac{1}{2}, 1\right], \quad \int_{0}^{T}|\nu(t)|^{2} d t>0, \\
& \text { or } \delta=\frac{1}{2}, \quad 4 \alpha(t) \geq|\nu(t)|^{2}, t \in[0, T], \quad \frac{\lambda}{2 T} \int_{0}^{T}\left(\int_{s}^{T} e^{-\int_{s}^{t}(\beta(u) / 2) d u} d t\right)^{2}|\nu(s)|^{2} d s \geq 1 .
\end{aligned}
$$

On the other hand,

$$
E\left[e^{\lambda \int_{0}^{T} r(t) d t}\right]<\infty
$$


provided

either $\delta=0$,

or $\quad \delta=\frac{1}{2}, \quad 4 \alpha(t) \leq|v(t)|^{2}, t \in[0, T], \quad \frac{\lambda T}{2} \int_{0}^{T} e^{\int_{0}^{s} \beta(u) d u}|v(s)|^{2} d s<1$.

Proof. For $\delta \in(1 / 2,1)$, by Itô's formula, we have (suppress argument $t$ )

$$
\begin{aligned}
d\left[r^{1-\delta}\right] & =\left\{(1-\delta) r^{-\delta}(\alpha-\beta r)+\frac{(1-\delta)(-\delta)}{2} r^{-\delta-1}|\nu|^{2} r^{2 \delta}\right\} d t+(1-\delta)\langle\nu, d W(t)\rangle \\
& =\left\{(1-\delta) \alpha r^{-\delta}-\frac{(1-\delta) \delta|\nu|^{2}}{2} r^{\delta-1}-(1-\delta) \beta r^{1-\delta}\right\} d t+(1-\delta)\langle\nu, d W(t)\rangle \\
& \equiv f(r) r^{1-\delta} d t+(1-\delta)\langle\nu, d W(t)\rangle,
\end{aligned}
$$

where

$$
f(r)=(1-\delta) \alpha r^{-1}-\frac{(1-\delta) \delta|\nu|^{2}}{2} r^{2(\delta-1)}-(1-\delta) \beta .
$$

Since $\delta>1 / 2$, one has $2(\delta-1)>-1$. Thus, $f(0+)=+\infty$ and $f(+\infty)=-(1-\delta) \beta$. Consequently, there exists an $\bar{f}(t) \in \mathbb{R}$ (deterministic) such that

$$
f(r(t)) \geq \bar{f}(t), \quad t \geq 0, \text { a.s. }
$$

Then (4.5) leads to

$$
d\left[r(t)^{1-\delta}\right] \geq \bar{f}(t) r(t)^{1-\delta} d t+(1-\delta)\langle v(t), d W(t)\rangle .
$$

We now take $\varphi(x)=\lambda|x|^{1 /(1-\delta)}$ for all $x \in \mathbb{R}$. Then one can apply Theorem 3.1(i) with $\gamma=1 /(1-\delta)>2$ and $c=\lambda$ to get $(4.1)$.

In the case that $\delta=1$, we have

$$
d[\log r]=\left\{\frac{\alpha-\beta r}{r}-\frac{1}{2}|\nu|^{2}\right\} d t+\langle\nu, d W(t)\rangle \geq-\left(\beta+\frac{|\nu|^{2}}{2}\right) d t+\langle\nu, d W(t)\rangle .
$$

Now, we take $\varphi(x)=\lambda e^{x}$. Then (3.3) holds for any $c>0$ and $\gamma>2$. Thus, (4.1) holds when the first line in (4.2) is assumed.

Next, we consider the case $\delta=1 / 2$. In this case, (4.5) yields (taking (4.2) into account)

$$
d\left[r^{1 / 2}\right]=\left(\frac{4 \alpha-|v|^{2}}{8} r^{-1 / 2}-\frac{\beta}{2} r^{1 / 2}\right) d t+\frac{1}{2}\langle v, d W(t)\rangle \geq-\frac{\beta}{2} r^{1 / 2} d t+\frac{1}{2}\langle v, d W(t)\rangle .
$$

Then (4.1) holds under (4.2) by using Theorem 3.1(i), with $\gamma=2$. 
On the other hand, for $\delta=1 / 2$, if (4.4) holds, we have, instead of (4.10), that

$$
d\left[r^{1 / 2}\right]=\left(\frac{4 \alpha-|\nu|^{2}}{8} r^{-1 / 2}-\frac{\beta}{2} r^{1 / 2}\right) d t+\frac{1}{2}\langle\nu, d W(t)\rangle \leq-\frac{\beta}{2} r^{1 / 2} d t+\frac{1}{2}\langle\nu, d W(t)\rangle .
$$

Then (4.3) follows from Theorem 3.1(ii) (with $\gamma=2$ ).

Finally, directly applying Theorem 3.1(ii) (with $\gamma=1$ ) to the case $\delta=0$, we can obtain (4.3).

By [7, pages 237-238], we know that when $d=1, \delta=1 / 2$, and $\alpha, \beta$, and $\nu$ are positive constants in (1.3), the following hold:

$$
\begin{aligned}
& \mathbf{P}\{\text { there are infinitely many } t>0 \text { for which } r(t)=0\}=1, \quad 2 \alpha<|\nu|^{2}, \\
& \mathbf{P}\{\text { there is at least one } t>0 \text { for which } r(t)=0\}=0, \quad 2 \alpha \geq|\nu|^{2} .
\end{aligned}
$$

Clearly, the second case in (4.12) should be more interesting than the first. But this implies the first condition in (4.3), which implies (4.1) when $T>0$ is large. Thus, Theorem 4.1 is basically a negative result in some sense.

We also note that if for the case $\delta \in(0,1 / 2),(1.3)$ admits a strong solution $r(\cdot)$ which is nonnegative valued, then we will still have (4.5) and (4.6). But now, since $\delta \in(0,1 / 2)$, $f(0+)=-\infty$ and $f(+\infty)=-(1-\delta) \beta$. Thus, instead of $(4.7)$, we have some deterministic $\bar{f}(t) \in \mathbb{R}$ such that

$$
f(r(t)) \leq \bar{f}(t), \quad t \geq 0, \text { a.s. }
$$

which leads to the following:

$$
d\left[r(t)^{1-\delta}\right] \leq \bar{f}(t) r(t)^{1-\delta} d t+(1-\delta)\langle\nu(t), d W(t)\rangle .
$$

Hence, taking $\varphi(x)=\lambda|x|^{1 /(1-\delta)}$ and applying Theorem 3.1 with $\gamma=1 /(1-\delta)<2$ and $c=\lambda$, we can also get (4.3). Of course, we have to assume the existence of a (nonnegative valued) strong solution $r(\cdot)$ to $(1.3)$.

We now consider (1.7). We have the following result.

TheOREM 4.2. Let (H1) hold, $\delta=0$ or $\delta \in[1 / 2,1]$. Let $r(\cdot)$ be the strong solution of $(1.3)$. Let $n=d$ with $\sigma(\cdot)^{-1}$ bounded and let $\theta(\cdot)$ be defined by (1.6). Then, for any $\lambda>0$,

$$
E\left[e^{\lambda \int_{0}^{T}|\theta(t)|^{2} d t}\right]=\infty
$$

provided

$$
\begin{aligned}
& \text { either } \delta \in\left(\frac{1}{2}, 1\right], \quad \int_{0}^{T}|\nu(t)|^{2} d t>0 \\
& \text { or } \delta=\frac{1}{2}, \quad 4 \alpha(t) \geq|\nu(t)|^{2}, t \in[0, T], \int_{0}^{T}|\nu(t)|^{2} d t>0 \\
& \text { or } \delta=0, \quad \frac{2 \lambda \inf _{t \in[0, T]}\left|\sigma(t)^{-1} \mathbf{1}\right|^{2}}{T} \int_{0}^{T}\left(\int_{s}^{T} e^{-\int_{s}^{t} \beta(u) d u} d t\right)^{2}|\nu(s)|^{2} d s>1 .
\end{aligned}
$$


On the other hand,

$$
E\left[e^{\lambda \int_{0}^{T}|\theta(t)|^{2} d t}\right]<\infty
$$

provided

$$
\delta=0, \quad 2 \lambda T \sup _{t \in[0, T]}\left|\sigma(t)^{-1} \mathbf{1}\right|^{2} \int_{0}^{T} e^{2 \int_{0}^{s} \beta(u) d u}|\nu(s)|^{2} d s<1 .
$$

Proof. We first note that

$$
\begin{aligned}
E\left[e^{\lambda \int_{0}^{T}|\theta(t)|^{2} d t}\right] & =E\left[e^{\lambda \int_{0}^{T}\left|\sigma(t)^{-1}[b(t)-r(t) \mathbf{1}]\right|^{2} d t}\right] \\
& \geq e^{-C_{\varepsilon}} E\left[e^{\lambda(1+\varepsilon) \int_{0}^{T}\left|\sigma(t)^{-1} \mathbf{1}\right|^{2} r(t)^{2} d t}\right] \\
& \geq e^{-C_{\varepsilon}} E\left[e^{\lambda(1+\varepsilon) \inf _{t \in[0, T]}\left|\sigma(t)^{-1} \mathbf{1}\right|^{2} \int_{0}^{T} r(t)^{2} d t}\right]
\end{aligned}
$$

Hence, when the first line in (4.16) holds with $\delta \in(1 / 2,1)$, one has (4.8). Thus, by taking $\varphi(x)=\lambda_{\varepsilon}|x|^{2 /(1-\delta)}$, with

$$
\lambda_{\varepsilon}=\lambda(1+\varepsilon) \inf _{t \in[0, T]}\left|\sigma(t)^{-1} \mathbf{1}\right|^{2}
$$

we can apply Theorem 3.1 with $\gamma=2 /(1-\delta)>2$ and $c=\lambda_{\varepsilon}$ to obtain (4.15). When the first line in (4.16) holds with $\delta=1$, one has (4.9). Hence, by taking $\varphi(x)=\lambda_{\varepsilon} e^{2 x}$, we see that (3.3) holds for any $c>0$ and $\gamma>2$. Thus, (4.15) holds. Now, for the case that the second line in (4.16) holds, we have (4.10). Thus, by taking $\varphi(x)=\lambda_{\varepsilon}|x|^{4}$, we obtain (4.16) by using Theorem 3.1(i) with $\gamma=4$. When the third line of (4.16) holds, we can apply Theorem 3.1(i) with $\varphi(x)=\lambda_{\varepsilon}|x|^{2}$ to get (4.15).

Finally, if (4.18) holds, we can apply Theorem 3.1(ii) to obtain (4.17).

The following result is concerned with (1.8).

Theorem 4.3. Let (H1) hold and $\delta=0$ or $\delta \in[1 / 2,1]$. Let there exist a $C^{1}$ function $k$ : $[0, \infty) \rightarrow(0, \infty)$ such that

$$
\begin{gathered}
k^{\prime}(t)+(2-\delta) \beta(t) k(t)>0, \quad \forall t>0, \\
\nu(t)=-k(t) \sigma(t)^{-1} \mathbf{1} .
\end{gathered}
$$

Let $r(\cdot)$ be the strong solution of (1.3). Then

$$
E\left[e^{\lambda \int_{0}^{T}\langle\theta(t), d W(t)\rangle}\right]=\infty
$$


306 Estimates on exponentials of solutions to SDEs

provided

$$
\begin{aligned}
& \text { either } \delta \in\left(\frac{1}{2}, 1\right], \quad \int_{0}^{T}|\nu(t)|^{2} d t>0 \\
& \text { or } \delta=\frac{1}{2}, \quad 4 \alpha(t) \geq|\nu(t)|^{2}, t \in[0, T], \int_{0}^{T}|\nu(t)|^{2} d t>0, \\
& \text { or } \delta=0, \quad \frac{\lambda}{k(T)} \int_{0}^{T} e^{-2 \int_{s}^{T} \beta(u) d u}|\nu(s)|^{2} d s>1 .
\end{aligned}
$$

On the other hand, if, instead of (4.21), one has

$$
\begin{gathered}
k^{\prime}(t)+2 \beta(t) k(t) \leq 0, \quad \forall t>0, \\
\nu(t)=-k(t) \sigma(t)^{-1} \mathbf{1},
\end{gathered}
$$

then

$$
E\left[e^{\lambda \int_{0}^{T}|\theta(t)|^{2} d t}\right]<\infty
$$

provided

$$
\delta=0, \quad \frac{\lambda}{k(T)} \int_{0}^{T} e^{2 \int_{0}^{s} \beta(u) d u}|\nu(s)|^{2} d s<1
$$

Proof. Consider (noting $\delta \in[0,1]$ and using (4.21))

$$
\begin{aligned}
d\left(r^{2-\delta}\right) & =(2-\delta) r^{1-\delta}(\alpha-\beta r) d t+\frac{(2-\delta)(1-\delta)|\nu|^{2}}{2} r^{\delta} d t+(2-\delta) r\langle\nu, d W(t)\rangle \\
& =(2-\delta)\left[\alpha r^{1-\delta}+\frac{(1-\delta)|\nu|^{2}}{2} r^{\delta}-\beta r^{2-\delta}\right] d t-(2-\delta) k r\left\langle\sigma^{-1} \mathbf{1}, d W(t)\right\rangle \\
& \leq(2-\delta)\left\{(\varepsilon-\beta) r^{2-\delta}+C_{\varepsilon}\right\} d t-(2-\delta) k r\left\langle\sigma^{-1} \mathbf{1}, d W(t)\right\rangle
\end{aligned}
$$

where $\varepsilon>0$ is small enough. Thus,

$$
\begin{aligned}
-r(t) & \left\langle\sigma(t)^{-1} \mathbf{1}, d W(t)\right\rangle \\
& \geq \frac{d\left[r(t)^{2-\delta}\right]}{(2-\delta) k(t)}-\frac{[\varepsilon-\beta(t)] r(t)^{2-\delta}+C_{\varepsilon}}{k(t)} d t \\
& =d\left(\frac{r(t)^{2-\delta}}{(2-\delta) k(t)}\right)+\left\{\frac{k^{\prime}(t)+(2-\delta)(\beta(t)-\varepsilon) k(t)}{(2-\delta) k(t)^{2}} r(t)^{2-\delta}-\frac{C_{\varepsilon}}{k(t)}\right\} d t \\
& \geq d\left(\frac{r(t)^{2-\delta}}{(2-\delta) k(t)}\right)-\frac{C_{\varepsilon}}{k(t)} d t, \quad t \in[0, T] .
\end{aligned}
$$


By (4.21), when we choose $\varepsilon>0$ small enough (which might be depending on $T$ ), the above inequality holds. Next, we assume that $\varepsilon \in(0, \lambda)$ (note (1.6)):

$$
\begin{aligned}
E\left[e^{-(\lambda-\varepsilon) \int_{0}^{T} r(t)\left\langle\sigma(t)^{-1} 1, d W(t)\right\rangle}\right] & \\
& =E\left[e^{(\lambda-\varepsilon) \int_{0}^{T}\langle\theta(t), d W(t)\rangle} e^{-(\lambda-\varepsilon) \int_{0}^{T}\left\langle\sigma(t)^{-1} b(t), d W(t)\right\rangle}\right] \\
& \leq\left\{E\left[e^{\lambda \int_{0}^{T}\langle\theta(t), d W(t)\rangle}\right]\right\}^{(\lambda-\varepsilon) / \lambda}\left\{E\left[e^{-((\lambda-\varepsilon) \lambda / \varepsilon) \int_{0}^{T}\left\langle\sigma(t)^{-1} b(t), d W(t)\right\rangle}\right]\right\}^{\varepsilon / \lambda} \\
& \leq C_{\varepsilon}\left\{E\left[e^{\lambda \int_{0}^{T}\langle\theta(t), d W(t)\rangle}\right]\right\}^{(\lambda-\varepsilon) / \lambda} .
\end{aligned}
$$

Hence, by (4.27),

$$
\begin{aligned}
\left\{E\left[e^{\lambda \int_{0}^{T}\langle\theta(t), d W(t)\rangle}\right]\right\}^{(\lambda-\varepsilon) / \lambda} & \geq C_{\varepsilon} E\left[e^{-(\lambda-\varepsilon) \int_{0}^{T} r(t)\left\langle\sigma(t)^{-1} 1, d W(t)\right\rangle}\right] \\
& \geq C_{\varepsilon} E\left[e^{(\lambda-\varepsilon) r(T)^{2-\delta /(2-\delta) k(T)}}\right] .
\end{aligned}
$$

Now, in the case that $\delta \in[1 / 2,1),(2-\delta) /(1-\delta)>2$. Thus, under (4.23), we have either (4.8) or (4.10). Hence, by taking $\varphi(x)=((\lambda-\varepsilon) /(2-\delta) k(T))|x|^{(2-\delta) /(1-\delta)}$, we can apply Theorem 3.1(i) with $\gamma=(2-\delta) /(1-\delta)>2$ and $c=(\lambda-\varepsilon) / 2(2-\delta) k(T)>0$ to get the right-hand side of (4.30) being infinite. The case $\delta=1$ can be treated similar to Theorem 4.1. In the case $\delta=0,(4.28)$ remains with $\delta=0$ and (4.30) becomes

$$
\left\{E\left[e^{\lambda \int_{0}^{T}\langle\theta(t), d W(t)\rangle}\right]\right\}^{(\lambda-\varepsilon) / \lambda} \geq C_{\varepsilon} E\left[e^{(\lambda-\varepsilon) r(T)^{2} / 2 k(T)}\right] .
$$

The right-hand side is infinite if the third line in (4.23) holds.

Finally, when (4.24) and (4.26) hold, we have (comparing with (4.27) and (4.28))

$$
\begin{aligned}
d\left(r^{2}\right) & =\left[2 r(\alpha-\beta r)+|v|^{2}\right] d t+2 r\langle\nu, d W(t)\rangle \\
& =\left[2 r(\alpha-\beta r)+|v|^{2}\right] d t-2 k r\left\langle\sigma^{-1} \mathbf{1}, d W(t)\right\rangle \\
& \geq-2 \beta r^{2} d t-2 k r\left\langle\sigma^{-1} \mathbf{1}, d W(t)\right\rangle .
\end{aligned}
$$

Thus,

$$
\begin{aligned}
-r(t)\left\langle\sigma(t)^{-1} \mathbf{1}, d W(t)\right\rangle & \leq \frac{d\left[r(t)^{2}\right]}{2 k(t)}+\frac{\beta(t) r(t)^{2}}{k(t)} d t \\
& =d\left(\frac{r(t)^{2}}{2 k(t)}\right)+\left\{\frac{k^{\prime}(t)+2 \beta(t) k(t)}{2 k(t)^{2}} r(t)^{2}\right\} d t \\
& \leq d\left(\frac{r(t)^{2}}{2 k(t)}\right), \quad t \in[0, T] .
\end{aligned}
$$


Then

$$
\begin{aligned}
& E\left[e^{\lambda \int_{0}^{T}\langle\theta(t), d W(t)\rangle}\right]=E\left[e^{\lambda \int_{0}^{T}\left\langle\sigma(t)^{-1}[b(t)-r(t) \mathbf{1}], d W(t)\right\rangle}\right] \\
& \leq C_{\varepsilon}\left\{E\left[e^{-(\lambda+\varepsilon) \int_{0}^{T} r(t)\left\langle\sigma(t)^{-1} 1, d W(t)\right\rangle}\right]\right\}^{\lambda /(\lambda+\varepsilon)} \\
& \leq C_{\varepsilon}\left\{E\left[e^{((\lambda+\varepsilon) / 2 k(T)) r(T)^{2}}\right]\right\}^{\lambda /(\lambda+\varepsilon)}<\infty
\end{aligned}
$$

provided (4.26) holds.

\section{Higher-dimensional cases}

In this section, we are going to present some results for higher dimensions. These results might be useful for problems involving multifactors (see $[1,5,6])$. We first make the following assumption.

$(\mathrm{H} 2)$ Let $b:[0, \infty) \times \mathbb{R}^{n} \rightarrow \mathbb{R}^{n}, \sigma:[0, \infty) \times \mathbb{R}^{n} \rightarrow \mathbb{R}^{n \times d}$ be given measurable functions such that for almost all $t \in[0, \infty), x \mapsto(b(t, x), \sigma(t, x))$ are continuous.

In what follows, we assume that the SDE (1.12) admits a unique strong solution $X(\cdot)$. We now state and prove our main result of this paper.

Theorem 5.1. Let $(H 2)$ hold and let $\delta \in[0,1]$ be a constant. Let $X(\cdot)$ be the strong solution of (1.12).

(i) Suppose there are functions $b_{0}^{\delta}(\cdot), b_{1}^{\delta}(\cdot) \in L_{\mathrm{loc}}^{1}(0, \infty ; \mathbb{R})$ such that

$$
\begin{gathered}
\frac{\langle x, b(t, x)\rangle}{|x|^{1+\delta}}+\frac{\operatorname{tr}\left[\sigma(t, x) \sigma(t, x)^{T}\right]}{2|x|^{1+\delta}}-\frac{(1+\delta)\left|\sigma(t, x)^{T} x\right|^{2}}{2|x|^{3+\delta}} \\
\geq b_{0}^{\delta}(t)+b_{1}^{\delta}(t)|x|^{1-\delta}, \quad t \geq 0, x \neq 0
\end{gathered}
$$

and there exists a nonnegative valued function $\delta_{0}(\cdot) \in L_{\text {loc }}^{2}(0, \infty ; \mathbb{R})$ such that

$$
\left|\sigma(t, x)^{T} x\right| \geq \delta_{0}(t)|x|^{1+\delta}, \quad \forall(t, x) \in[0, \infty) \times \mathbb{R}^{n} .
$$

Suppose $\varphi: \mathbb{R} \rightarrow[0, \infty)$ is continuous and satisfies the following:

$$
\varliminf_{|x| \rightarrow \infty} \frac{\varphi(x)}{|x|^{\gamma}}>c,
$$

for some constants $\gamma, c>0$. Then

$$
E\left[e^{\varphi(X(t))}\right]=\infty
$$

provided

$$
\begin{aligned}
& \text { either } \quad \gamma>2(1-\delta), \quad \int_{0}^{t} \delta_{0}(s)^{2} d s>0, \\
& \text { or } \quad \gamma=2(1-\delta), \quad 2 c(1-\delta)^{2} \int_{0}^{t} e^{-2(1-\delta) \int_{0}^{s} b_{1}^{\delta}(u) d u} \delta_{0}(s)^{2} d s>1,
\end{aligned}
$$


and

$$
E\left[e^{\int_{0}^{T} \varphi(X(t)) d t}\right]=\infty
$$

provided

$$
\begin{aligned}
& \text { either } \quad \gamma>2(1-\delta), \quad \int_{0}^{t} \delta_{0}(s)^{2} d s>1 \\
& \text { or } \quad \gamma=2(1-\delta), \quad \frac{2 c(1-\delta)^{2}}{T} \int_{0}^{T}\left(\int_{s}^{T} e^{(1-\delta) \int_{s}^{t} b_{1}^{\delta}(u) d u}\right)^{2} \delta_{0}(s)^{2} d s>1 .
\end{aligned}
$$

In addition, for the case $\gamma=2(1-\delta)$, if $b_{0}^{\delta}(t) \geq 0$, for all $t \in[0, \infty)$, then "> " in the second lines in (5.5) and (5.7) can be replaced by " $\geq$ ".

(ii) Suppose there are $\bar{b}_{0}^{\delta}(\cdot), \bar{b}_{1}^{\delta}(\cdot) \in L_{\text {loc }}^{1}(0, \infty ; \mathbb{R})$ such that

$$
\begin{gathered}
\frac{\langle x, b(t, x)\rangle}{|x|^{1+\delta}}+\frac{\operatorname{tr}\left[\sigma(t, x) \sigma(t, x)^{T}\right]}{2|x|^{1+\delta}}-\frac{(1+\delta)\left|\sigma(t, x)^{T} x\right|^{2}}{2|x|^{3+\delta}} \\
\leq \bar{b}_{0}^{\delta}(t)+\bar{b}_{1}^{\delta}(t)|x|^{1-\delta}, \quad t \geq 0, x \neq 0,
\end{gathered}
$$

and there exists a function $L_{0}(\cdot) \in L_{\mathrm{loc}}^{2}(0, \infty ; \mathbb{R})$ such that

$$
\left|\sigma(t, x)^{T} x\right| \leq L_{0}(t)|x|^{1+\delta}, \quad \forall(t, x) \in[0, \infty) \times \mathbb{R}^{n} .
$$

Suppose $\varphi: \mathbb{R}^{n} \rightarrow \mathbb{R}$ is continuous and satisfies the following:

$$
\lim _{|x| \rightarrow \infty} \frac{\varphi(x)}{|x|^{\gamma}}<c,
$$

for some constants $\gamma \geq 0$ and $c>0$. Then

$$
E\left[\sup _{t \in[0, T]} e^{\varphi(X(t))}\right]<\infty
$$

provided

$$
\begin{aligned}
& \text { either } 0 \leq \gamma<2(1-\delta) \text {, } \\
& \text { or } \quad \gamma=2(1-\delta), \quad 2 c(1-\delta)^{2} \int_{0}^{T} e^{2(1-\delta) \int_{s}^{T} \hat{b}_{1}^{\delta}(u) d u} L_{0}(s)^{2} d s<1,
\end{aligned}
$$

and

$$
E\left[e^{\int_{0}^{T} \varphi(X(t)) d t}\right]<\infty
$$

provided

$$
\begin{aligned}
& \text { either } \quad 0 \leq \gamma<2(1-\delta) \text {, } \\
& \text { or } \quad \gamma=2(1-\delta), \quad 2 c(1-\delta)^{2} T \int_{0}^{T} e^{2(1-\delta) \int_{s}^{T} b_{1}^{\delta}(u) d u} L_{0}(s)^{2} d s<1 .
\end{aligned}
$$


Proof. (i) For any $\varepsilon>0$ and $x \in \mathbb{R}^{n}$, define $\langle x\rangle_{\varepsilon} \triangleq \sqrt{\varepsilon+|x|^{2}}$. Then, for any $\delta \in \mathbb{R}, \delta \neq 1$, the following hold:

$$
\begin{aligned}
{\left[\langle x\rangle_{\varepsilon}^{1-\delta}\right]_{x} } & =(1-\delta)\langle x\rangle_{\varepsilon}^{-1-\delta} x, \\
{\left[\langle x\rangle_{\varepsilon}^{1-\delta}\right]_{x x} } & =(1-\delta)\langle x\rangle_{\varepsilon}^{-1-\delta} I-(1-\delta)(1+\delta)\langle x\rangle_{\varepsilon}^{-3-\delta} x x^{T}
\end{aligned}
$$

Now, let $X(\cdot)$ be the strong solution of (1.12) and let $\delta \in[0,1)$. Applying Itô's formula to $\langle X(\cdot)\rangle_{\varepsilon}^{1-\delta}$, we obtain the following:

$$
\begin{aligned}
d\left[\langle X(t)\rangle_{\varepsilon}^{1-\delta}\right]= & \{1-\delta)\langle X(t)\rangle_{\varepsilon}^{-1-\delta}\langle X(t), b(t, X(t))\rangle \\
& +\frac{1-\delta}{2}\langle X(t)\rangle_{\varepsilon}^{-1-\delta} \operatorname{tr}\left[\sigma(t, X(t)) \sigma(t, X(t))^{T}\right] \\
& \left.-\frac{(1-\delta)(1+\delta)}{2}\langle X(t)\rangle_{\varepsilon}^{-3-\delta}\left|\sigma(t, X(t))^{T} X(t)\right|^{2}\right\} d t \\
& +(1-\delta)\langle X(t)\rangle_{\varepsilon}^{-1-\delta}\left\langle\sigma(t, X(t))^{T} X(t), d W(t)\right\rangle \\
\equiv & b_{\varepsilon}^{\delta}(t, X(t)) d t+\left\langle\sigma_{\varepsilon}^{\delta}(t, X(t)), d W(t)\right\rangle,
\end{aligned}
$$

where

$$
\begin{gathered}
b_{\varepsilon}^{\delta}(t, x)=(1-\delta)\left\{\langle x\rangle_{\varepsilon}^{-1-\delta}\langle x, b(t, x)\rangle+\frac{1}{2}\langle x\rangle_{\varepsilon}^{-1-\delta} \operatorname{tr}\left[\sigma(t, x) \sigma(t, x)^{T}\right]\right. \\
\left.-\frac{1+\delta}{2}\langle x\rangle_{\varepsilon}^{-3-\delta}\left|\sigma(t, x)^{T} x\right|^{2}\right\} \\
\sigma_{\varepsilon}^{\delta}(t, x)=(1-\delta)\langle x\rangle_{\varepsilon}^{-1-\delta} \sigma(t, x)^{T} x .
\end{gathered}
$$

By (5.1), we have

$$
\begin{aligned}
\lim _{\varepsilon \rightarrow 0} b_{\varepsilon}^{\delta}(t, x) & =(1-\delta)\left\{\frac{\langle x, b(t, x)\rangle}{|x|^{1+\delta}}+\frac{\operatorname{tr}\left[\sigma(t, x) \sigma(t, x)^{T}\right]}{2|x|^{1+\delta}}-\frac{(1+\delta)\left|\sigma(t, x)^{T} x\right|^{2}}{2|x|^{3+\delta}}\right\} \\
& \geq(1-\delta)\left\{b_{0}^{\delta}(t)+b_{1}^{\delta}(t)|x|^{1-\delta}\right\}, \quad t \geq 0, x \neq 0 \\
\lim _{\varepsilon \rightarrow 0} \sigma_{\varepsilon}^{\delta}(t, x) & =(1-\delta) \frac{\sigma(t, x)^{T} x}{|x|^{1+\delta}} \equiv(1-\delta) \sigma^{\delta}(t, x), \quad t \geq 0, x \neq 0 .
\end{aligned}
$$

Thus, we have

$$
d\left[|X(t)|^{1-\delta}\right] \geq(1-\delta)\left\{b_{0}^{\delta}(t)+b_{1}^{\delta}|X(t)|^{1-\delta}\right\} d t+(1-\delta)\left\langle\sigma^{\delta}(t, X(t)), d W(t)\right\rangle
$$

Then, by Theorem 3.1, we obtain the conclusions in (i) for $\delta \in[0,1$ ). 
We now look at the case $\delta=1$. For this case, we only need to show that (5.4) and (5.6) hold if the first lines in (5.5) and (5.7) hold, respectively. To this end, we note that when $\delta=1$, instead of (5.15), one has

$$
\begin{aligned}
{\left[\ln \langle x\rangle_{\varepsilon}\right]_{x} } & =\langle x\rangle_{\varepsilon}^{-2} x, \\
{\left[\ln \langle x\rangle_{\varepsilon}\right]_{x x} } & =\langle x\rangle_{\varepsilon}^{-2} I-2\langle x\rangle_{\varepsilon}^{-4} x x^{T}
\end{aligned}
$$

Thus, applying Itô's formula to $\ln \langle X(t)\rangle_{\varepsilon}$, we have the following:

$$
\begin{aligned}
d\left[\ln \langle X(t)\rangle_{\varepsilon}\right]= & {\left[\frac{\langle X(t), b(t, X(t))\rangle}{\langle X(t)\rangle_{\varepsilon}^{2}}+\frac{\operatorname{tr}\left[\sigma(t, X(t)) \sigma(t, X(t))^{T}\right]}{2\langle X(t)\rangle_{\varepsilon}^{2}}\right.} \\
& \left.-\frac{\left|\sigma(t, X(t))^{T} X(t)\right|^{2}}{\langle X(t)\rangle_{\varepsilon}^{4}}\right] d t+\frac{\left\langle\sigma(t, X(t))^{T} X(t), d W(t)\right\rangle}{\langle X(t)\rangle_{\varepsilon}^{2}} \\
\equiv & b_{\varepsilon}^{1}(t, X(t)) d t+\left\langle\sigma_{\varepsilon}^{1}(t, X(t)), d W(t)\right\rangle,
\end{aligned}
$$

where

$$
\begin{gathered}
b_{\varepsilon}^{1}(t, x) \triangleq \frac{\langle x, b(t, x)\rangle}{\langle x\rangle_{\varepsilon}^{2}}+\frac{\operatorname{tr}\left[\sigma(t, x) \sigma(t, x)^{T}\right]}{2\langle x\rangle_{\varepsilon}^{2}}-\frac{\left|\sigma(t, x)^{T} x\right|^{2}}{\langle x\rangle_{\varepsilon}^{4}}, \\
\sigma_{\varepsilon}^{1}(t, x)=\frac{\sigma(t, x)^{T} x}{\langle x\rangle_{\varepsilon}^{2}}
\end{gathered}
$$

Thus, by (5.1) (with $\delta=1$ ), we have

$$
\begin{aligned}
\lim _{\varepsilon \rightarrow 0} b_{\varepsilon}^{1}(t, x) & =\frac{\langle x, b(t, x)\rangle}{|x|^{2}}+\frac{\operatorname{tr}\left[\sigma(t, x) \sigma(t, x)^{T}\right]}{2|x|^{2}}-\frac{\left|\sigma(t, x)^{T} x\right|^{2}}{|x|^{4}} \\
& \geq b_{0}^{1}(t)+b_{1}^{1}(t) \equiv \tilde{b}(t), \quad t \geq 0, x \neq 0 .
\end{aligned}
$$

Also, we let

$$
\tilde{\sigma}(t, x)= \begin{cases}\frac{\sigma(t, x)^{T} x}{|x|^{2}}, & x \neq 0, \\ 0, & x=0 .\end{cases}
$$

Then it follows from (5.21) that

$$
d(\ln |X(t)|) \geq \tilde{b}(t) d t+\langle\tilde{\sigma}(t, X(t)), d W(t)\rangle .
$$

Consequently,

$$
|X(t)| \geq\left|X_{0}\right| e^{\int_{0}^{t} \tilde{b}(s) d s} e^{\int_{0}^{t}\langle\tilde{\sigma}(s, X(s)), d W(s)\rangle}, \quad t \in[0, \infty) .
$$


Now, by (5.3), there exists a constant $R>0$ such that

$$
\begin{aligned}
\varphi(X(t)) & \geq c|X(t)|^{\gamma}-R \\
& \geq c\left|X_{0}\right|^{\gamma} e^{\gamma \int_{0}^{t} \tilde{b}(s) d s} e^{\gamma \int_{0}^{t}\langle\tilde{\sigma}(s, X(s)), d W(s)\rangle}-R \\
& \equiv C(t) e^{\gamma \int_{0}^{t}\langle\tilde{\sigma}(s, X(s)), d W(s)\rangle}-R, \quad t \in[0, \infty),
\end{aligned}
$$

where $C(\cdot) \in L_{\text {loc }}^{\infty}(0, \infty ; \mathbb{R})$ is taking positive values. Consequently, when $\int_{0}^{t} \delta_{0}(s)^{2} d s>0$, by Lemma 2.1, we have

$$
E\left[e^{\varphi(X(t))}\right] \geq E\left[e^{C(t) e^{\gamma \int_{0}^{t}\langle\tilde{\sigma}(s, X(s)), d W(s)\rangle}-R}\right] \geq e^{-R} E\left[e^{\left(C(t) \gamma^{4} / 4 !\right)\left|\int_{0}^{t}\{\tilde{\sigma}(s, X(s)), d W(s)\rangle\right|^{4}}\right]=\infty .
$$

This proves (i).

(ii) Again, for the case $\delta \in[0,1)$, instead of (5.19), we have

$$
d\left[|X(t)|^{1-\delta}\right] \leq(1-\delta)\left\{\bar{b}_{0}^{\delta}(t)+\bar{b}_{1}^{\delta}|X(t)|^{1-\delta}\right\} d t+(1-\delta)\left\langle\sigma^{\delta}(t, X(t)), d W(t)\right\rangle .
$$

Then, by Theorem 3.1, we obtain the conclusions in (ii) for $\delta \in[0,1)$.

Finally, for $\delta=1$, it is necessary that $\gamma=0$. Thus, from (5.12) or $(5.14), \varphi(\cdot)$ is necessarily bounded. Hence, (5.11) and (5.13) hold.

We now look at when (1.15) holds. This problem is a little more complicated. We present an example first.

Example 5.2. Let

$$
X(t)=W(t), \quad t \geq 0 .
$$

Let $\gamma>1$. Applying Itô's formula to $e^{-t}|X(t)|^{\gamma+1}$, one has

$$
\begin{aligned}
d\left[e^{-t}|X(t)|^{\gamma+1}\right]= & (\gamma+1) e^{-t}|X(t)|^{\gamma-1}\langle X(t), d W(t)\rangle \\
& +e^{-t}\left[-|X(t)|^{\gamma+1}+\frac{(\gamma+1)(\gamma-1)}{2}|X(t)|^{\gamma-1}\right] d t \\
\leq & \langle\mu(t, X(t)), d W(t)\rangle+C d t,
\end{aligned}
$$

where

$$
\mu(t, x)=(\gamma+1) e^{-t}|x|^{\gamma-1} x, \quad(t, x) \in[0, \infty) \times \mathbb{R}^{d} .
$$

Then

$$
E\left[e^{\int_{0}^{t}\langle\mu(s, X(s)), d W(s)\rangle}\right] \geq C(t) E\left[e^{e^{-t}|W(t)|^{\gamma+1}}\right]=\infty
$$

provided $t>0$ (by Corollary 2.2 and noting $\gamma>1$ ). On the other hand, if we take

$$
\tilde{\mu}(t, x)=-(\gamma+1) e^{t}|x|^{\gamma-1} x \equiv-\mu(t, x), \quad(t, x) \in[0, \infty) \times \mathbb{R}^{d},
$$


then

$$
E\left[\sup _{t \in[0, T]} e^{\int_{0}^{t}\langle\tilde{\mu}(s, X(s)), d W(s)\rangle}\right] \leq C(T) E\left[\sup _{t \in[0, T]} e^{-e^{t}|W(t)|^{\mid+1}}\right]<\infty,
$$

for any $T \geq 0$.

The above example tells that not only the growth of $x \mapsto|\mu(t, x)|($ as $|x| \rightarrow \infty)$ plays the role, but also the "direction" of $\mu(t, x)$ matters. The following result is concerned with (1.15) in a general manner.

TheOREM 5.3. Let (H2) hold and let $\delta \in[0,1]$ be a constant. Let $X(\cdot)$ be the strong solution of (1.12).

(i) Let (5.1) and (5.2) hold for some functions $b_{0}^{\delta}(\cdot), b_{1}^{\delta}(\cdot) \in L_{\mathrm{loc}}^{1}(0, \infty ; \mathbb{R})$ and $\delta_{0}(\cdot) \in$ $L_{\text {loc }}^{2}(0, \infty ; \mathbb{R})$. Suppose $\mu:[0, \infty) \times \mathbb{R}^{n} \rightarrow \mathbb{R}^{d}$ is continuous such that

$$
\mu(t, x)=\sigma(t, x)^{T} \psi_{x}(t, x), \quad \forall(t, x) \in[0, \infty) \times \mathbb{R}^{n},
$$

for some $\psi(\cdot, \cdot)$ satisfying

$$
\begin{aligned}
\psi_{t}(t, x)+\left\langle\psi_{x}(t, x), b(t, x)\right\rangle+\frac{1}{2} \operatorname{tr}\left[\psi_{x x}(t, x) \sigma(t, x) \sigma(t, x)^{T}\right] \leq a(t) & \forall(t, x) \in[0, \infty) \times \mathbb{R}^{n}, \\
\lim _{|x| \rightarrow \infty} \inf _{t \in[0, T]} \frac{\psi(t, x)}{|x|^{\gamma}}>c, & \forall T>0,
\end{aligned}
$$

for some $\gamma, c>0$ and some $a(\cdot) \in L_{\mathrm{loc}}^{1}(0, \infty ; \mathbb{R})$. Then

$$
E\left[e^{\int_{0}^{t}\langle\mu(s, X(s)), d W(s)\rangle}\right]=\infty
$$

provided (5.5) holds.

(ii) Let (5.8) and (5.9) hold for some functions $\bar{b}_{0}^{\delta}(\cdot), \bar{b}_{1}^{\delta}(\cdot) \in L_{\mathrm{loc}}^{1}(0, \infty ; \mathbb{R})$ and $L_{0}(\cdot) \in$ $L_{\text {loc }}^{2}(0, \infty ; \mathbb{R})$. Suppose $\mu:[0, \infty) \times \mathbb{R}^{n} \rightarrow \mathbb{R}^{d}$ is continuous such that (5.36) holds for some $\psi(\cdot, \cdot)$ satisfying

$$
\begin{array}{r}
\psi_{t}(t, x)+\left\langle\psi_{x}(t, x), b(t, x)\right\rangle+\frac{1}{2} \operatorname{tr}\left[\psi_{x x}(t, x) \sigma(t, x) \sigma(t, x)^{T}\right] \geq \bar{a}(t) \\
\forall(t, x) \in[0, \infty) \times \mathbb{R}^{n}, \\
\lim _{|x| \rightarrow \infty} \sup _{t \in[0, T]} \frac{\psi(t, x)}{|x|^{\gamma}}<c, \quad \forall T>0,
\end{array}
$$

for some $\gamma, c>0$ and some $\bar{a}(\cdot) \in L_{\text {loc }}^{1}(0, \infty ; \mathbb{R})$. Then

$$
E\left[\sup _{t \in[0, T]} e^{\int_{0}^{t}\langle\mu(s, X(s)), d W(s)\rangle}\right]<\infty
$$

provided (5.12) holds. 
314 Estimates on exponentials of solutions to SDEs

(iii) Let (5.8) and (5.9) hold for some functions $\bar{b}_{0}^{\delta}(\cdot), \bar{b}_{1}^{\delta}(\cdot) \in L_{\mathrm{loc}}^{1}(0, \infty ; \mathbb{R})$ and $L_{0}(\cdot) \in$ $L_{\text {loc }}^{2}(0, \infty ; \mathbb{R})$. Suppose $\mu:[0, \infty) \times \mathbb{R}^{n} \rightarrow \mathbb{R}^{d}$ such that

$$
|\mu(t, x)| \leq L\left(1+|x|^{\gamma}\right), \quad \forall(t, x) \in[0, \infty) \times \mathbb{R}^{n},
$$

with $\gamma, L>0$. Then (5.42) holds provided

$$
\begin{aligned}
& \text { either } \quad 0 \leq \gamma<1-\delta \\
& \text { or } \quad \gamma=1-\delta, \quad 4 L^{2}(1-\delta)^{2} T \int_{0}^{T} e^{2(1-\delta) \int_{s}^{T} \bar{b}_{1}^{\delta}(u) d u} L_{0}(s)^{2} d s<1 .
\end{aligned}
$$

Proof. (i) Applying Itô's formula to $\psi(\cdot, X(\cdot))$, we have

$$
\begin{aligned}
d[\psi(t, X(t))]= & \left\{\psi_{t}(t, X(t))+\left\langle\psi_{x}(t, X(t)), b(t, X(t))\right\rangle\right. \\
& \left.+\frac{1}{2} \operatorname{tr}\left[\psi_{x x}(t, X(t)) \sigma(t, X(t)) \sigma(t, X(t))^{T}\right]\right\} d t \\
& +\left\langle\sigma(t, X(t))^{T} \psi_{x}(t, X(t)), d W(t)\right\rangle \\
\leq & a(t) d t+\langle\mu(t, X(t)), d W(t)\rangle .
\end{aligned}
$$

Consequently, noting (5.38),

$$
c|X(t)|^{\gamma}-R \leq \psi(t, X(t)) \leq \psi\left(0, X_{0}\right)+\int_{0}^{t} a(s) d s+\int_{0}^{t}\langle\mu(s, X(s)), d W(s)\rangle .
$$

Thus, by Theorem 5.1, one obtains

$$
E\left[e^{\int_{0}^{t}\langle\mu(t, X(t)), d W(t)\rangle}\right] \geq E\left[e^{c|X(t)|^{\gamma}-R-\psi\left(0, X_{0}\right)-\int_{0}^{t} a(s) d s}\right]=\infty
$$

if (5.5) holds.

(ii) Applying Itô's formula to $\psi(\cdot, X(\cdot))$, we have

$$
\begin{aligned}
d[\psi(t, X(t))]= & \left\{\psi_{t}(t, X(t))+\left\langle\psi_{x}(t, X(t)), b(t, X(t))\right\rangle\right. \\
& \left.+\frac{1}{2} \operatorname{tr}\left[\psi_{x x}(t, X(t)) \sigma(t, X(t)) \sigma(t, X(t))^{T}\right]\right\} d t \\
& +\left\langle\sigma(t, X(t))^{T} \psi_{x}(t, X(t)), d W(t)\right\rangle \\
\geq & \bar{a}(t) d t+\langle\mu(t, X(t)), d W(t)\rangle .
\end{aligned}
$$

Consequently, noting (5.38),

$$
c|X(t)|^{\gamma}-R \geq \psi(t, X(t)) \geq \psi\left(0, X_{0}\right)+\int_{0}^{t} \bar{a}(s) d s+\int_{0}^{t}\langle\mu(s, X(s)), d W(s)\rangle .
$$


Thus, by Theorem 5.1, one obtains

$$
E\left[\sup _{t \in[0, T]} e^{\int_{0}^{t}\langle\mu(t, X(t)), d W(t)\rangle}\right] \leq E\left[\sup _{t \in[0, T]} e^{c|X(t)|^{\gamma}-R-\psi\left(0, X_{0}\right)-\int_{0}^{t} \bar{a}(s) d s}\right]<\infty
$$

if (5.12) holds.

(iii) Note that when $0 \leq \gamma<1-\delta$, by Theorem 5.1 , we have

$$
\begin{aligned}
& E\left[\sup _{t \in[0, T]} e^{\int_{0}^{t}\langle\mu(s, X(s)), d W(s)\rangle}\right] \\
&=E\left[\sup _{t \in[0, T]} e^{(1 / 2) \int_{0}^{t}\langle 2 \mu(s, X(s)), d W(s)\rangle-(1 / 4) \int_{0}^{t}|2 \mu(s, X(s))|^{2} d s} e^{\left.(1 / 4) \int_{0}^{t}|2 \mu(s, X(s))|^{2} d s\right]}\right. \\
& \leq\left\{E \left[\sup _{t \in[0, T]} e^{\left.\left.\int_{0}^{t}\langle 2 \mu(s, X(s)), d W(s)\rangle-(1 / 2) \int_{0}^{t}|2 \mu(s, X(s))|^{2} d s\right]\right\}^{1 / 2}}\right.\right. \\
& \cdot\left\{E\left[\sup _{t \in[0, T]} e^{2 \int_{0}^{t}|\mu(s, X(s))|^{2} d s}\right]\right\}^{1 / 2} \\
& \leq\left\{E\left[\sup _{t \in[0, T]} e^{2 \int_{0}^{t}|\mu(s, X(s))|^{2} d s}\right]\right\}^{1 / 2} \\
& \leq C_{\varepsilon} E\left[\sup _{t \in[0, T]} e^{2 L^{2}(1+\varepsilon) \int_{0}^{T}|X(s)|^{2 \gamma} d s}\right]<\infty
\end{aligned}
$$

for any $\varepsilon>0$. In the case $\gamma=1-\delta$, the above remains true if the second line in (5.44) holds.

Note that in Example 5.2, if we take

$$
\psi(t, x)=e^{-t}|x|^{\gamma+1}, \quad(t, x) \in[0, \infty) \times \mathbb{R}
$$

then (5.37) holds, which leads to (5.31) and (5.33). On the other hand, if we take

$$
\psi(t, x)=-e^{-t}|x|^{\gamma+1}, \quad(t, x) \in[0, \infty) \times \mathbb{R},
$$

then (5.40) holds, which leads to (5.35). We point out that $\gamma>1$ is arbitrary here, but the signs in (5.51) and (5.52) are crucial, whereas Theorem 5.3(iii) says that when $\gamma$ is small, the "direction" of $\mu(\cdot, \cdot)$ is irrelevant.

\section{Acknowledgments}

This work was supported in part by the NSFC under Grant 10131030, the Chinese Ministry of Education Science Foundation under Grant 2000024605, the Cheung Kong Scholars Programme, and Shanghai Commission of Science and Technology under Grant 02DJ14063. 


\section{References}

[1] T. R. Bielecki, S. Pliska, and J. Yong, Optimal investment decisions for a portfolio with a rolling horizon bond and a discount bond, submitted to Int. J. Theoretical \& Appl. Finance.

[2] V. V. Buldygin and Yu. V. Kozachenko, Metric Characterization of Random Variables and Random Processes, Translations of Mathematical Monographs, vol. 188, American Mathematical Society, Rhode Island, 2000.

[3] J. C. Cox, J. E. Ingersoll Jr., and S. A. Ross, A theory of the term structure of interest rates, Econometrica 53 (1985), no. 2, 385-407.

[4] F. Delbaen and W. Schachermayer, A general version of the fundamental theorem of asset pricing, Math. Ann. 300 (1994), no. 3, 463-520.

[5] D. Duffie, D. Filipović, and W. Schachermayer, Affine processes and applications in finance, Ann. Appl. Probab. 13 (2003), no. 3, 984-1053.

[6] D. Duffie and R. Kan, A yield-factor model of interest rates, Math. Finance 6 (1996), no. 4, 379406.

[7] R. J. Elliott and P. E. Kopp, Mathematics of Financial Markets, Springer Finance, SpringerVerlag, New York, 1999.

[8] J. Hull and A. White, Pricing interest-rate derivative securities, Rev. Finan. Stud. 3 (1990), no. 4, 573-592.

[9] N. Ikeda and S. Watanabe, Stochastic Differential Equations and Diffusion Processes, NorthHolland Mathematical Library, vol. 24, North-Holland Publishing, Amsterdam, 1981.

[10] I. Karatzas and S. E. Shreve, Brownian Motion and Stochastic Calculus, Graduate Texts in Mathematics, vol. 113, Springer-Verlag, New York, 1988.

[11] Methods of Mathematical Finance, Applications of Mathematics (New York), vol. 39, Springer-Verlag, New York, 1998.

[12] N. Kazamaki, Continuous Exponential Martingales and BMO, Lecture Notes in Mathematics, vol. 1579, Springer-Verlag, Berlin, 1994.

[13] M. Musiela and M. Rutkowski, Martingale Methods in Financial Modelling, Applications of Mathematics (New York), vol. 36, Springer-Verlag, Berlin, 1997.

[14] A. A. Novikov, On an identity for stochastic integrals, Theory Probab. Appl. 17 (1972), 717-720.

[15] P. Protter, Stochastic Integration and Differential Equations, Applications of Mathematics (New York), vol. 21, Springer-Verlag, Berlin, 1990.

[16] D. Revuz and M. Yor, Continuous Martingales and Brownian Motion, 3rd ed., Grundlehren der mathematischen Wissenschaften, vol. 293, Springer-Verlag, Berlin, 1999.

[17] O. A. Vasicek, An equilibrium characterization of term structure, J. Finan. Econ. 5 (1977), 177188.

[18] J. Yong, European-type contingent claims in an incomplete market with constrained wealth and portfolio, Math. Finance 9 (1999), no. 4, 387-412.

[19] M. Yor, Exponential Functionals of Brownian Motion and Related Processes, Springer Finance, Springer-Verlag, Berlin, 2001.

Jiongmin Yong: Institute of Mathematical Finance, Fudan University, Shanghai 200433, China

Current address: Department of Mathematics, University of Central Florida, Orlando, FL 32816, USA

E-mail address: jyong@mail.ucf.edu 


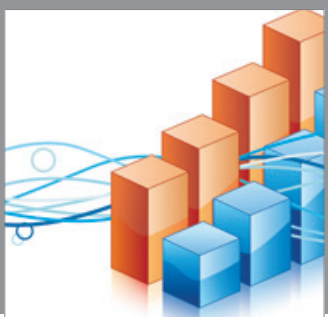

Advances in

Operations Research

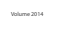

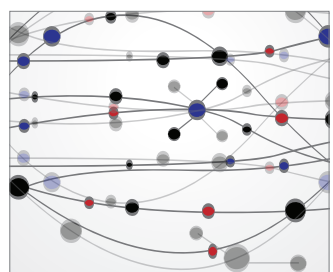

\section{The Scientific} World Journal
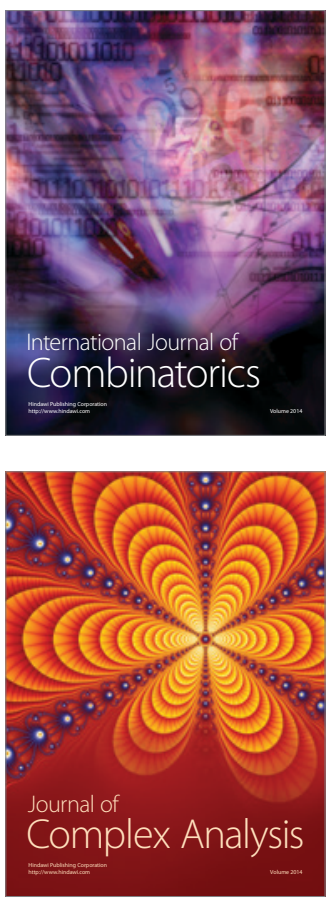

International Journal of

Mathematics and

Mathematical

Sciences
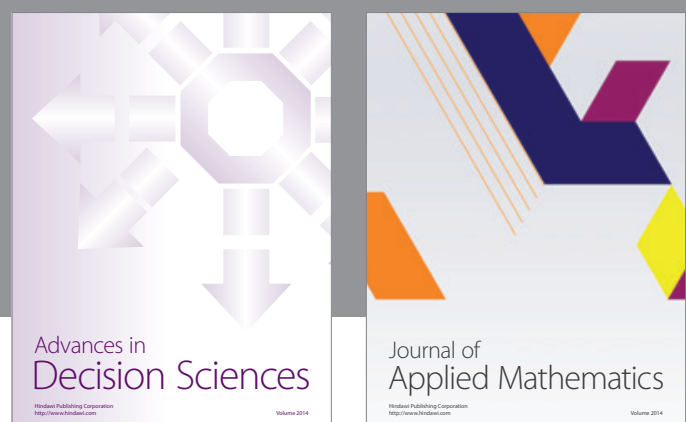

Journal of

Applied Mathematics
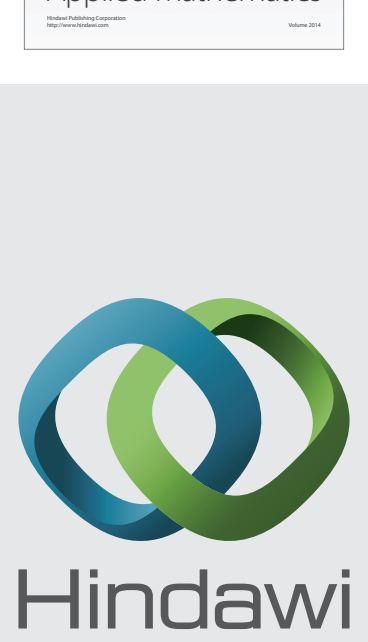

Submit your manuscripts at http://www.hindawi.com
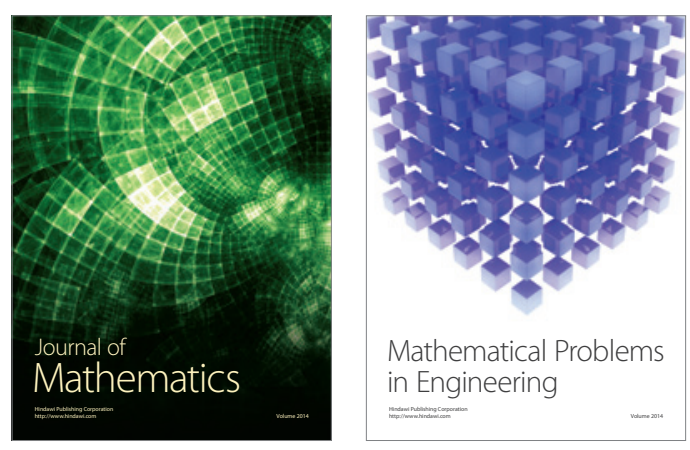

Mathematical Problems in Engineering
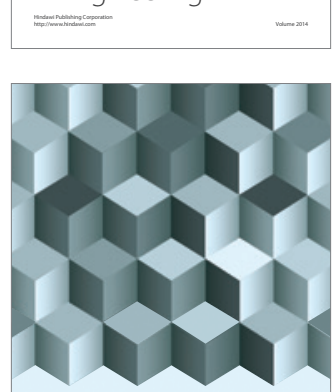

Journal of

Function Spaces
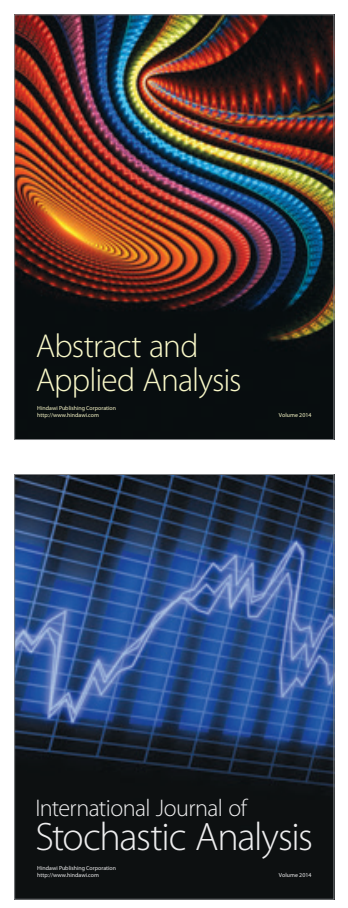

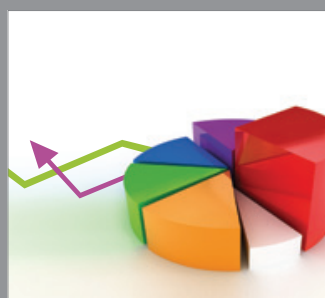

ournal of

Probability and Statistics

Promensencen
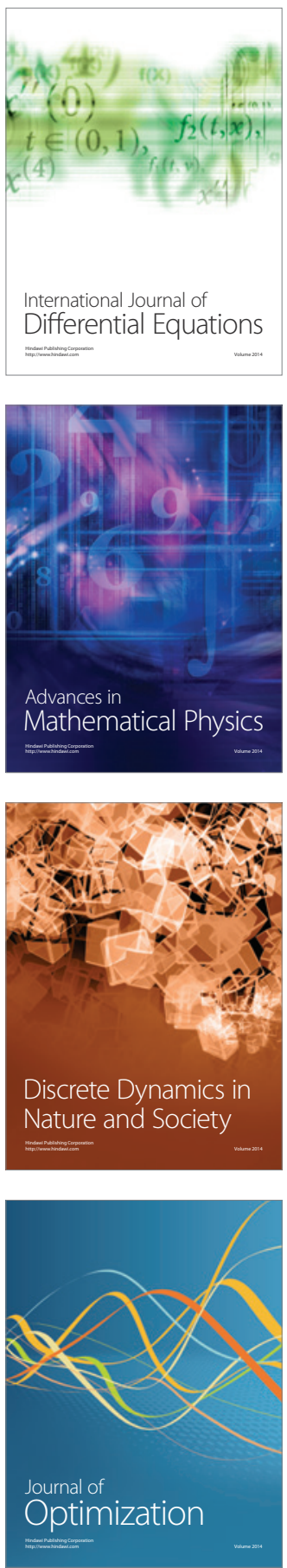